(c) The Authors 2015. This is an Open Access article, distributed under the terms of the Creative Commons Attribution licence (http:// creativecommons.org/licenses/by/3.0/), which permits unrestricted re-use, distribution, and reproduction in any medium, provided the original work is properly cited.

\title{
Understanding meal patterns: definitions, methodology and impact on nutrient intake and diet quality
}

\author{
Rebecca M. Leech*, Anthony Worsley, Anna Timperio and Sarah A. McNaughton \\ Centre for Physical Activity and Nutrition Research (C-PAN), School of Exercise and Nutrition Sciences, Deakin University, \\ 221 Burwood Highway, Burwood, VIC 3125, Australia
}

\section{Abstract}

Traditionally, nutrition research has focused on individual nutrients, and more recently dietary patterns. However, there has been relatively little focus on dietary intake at the level of a 'meal'. The purpose of the present paper was to review the literature on adults' meal patterns, including how meal patterns have previously been defined and their associations with nutrient intakes and diet quality. For this narrative literature review, a comprehensive search of electronic databases was undertaken to identify studies in adults aged $\geq 19$ years that have investigated meal patterns and their association with nutrient intakes and/or diet quality. To date, different approaches have been used to define meals with little investigation of how these definitions influence the characterisation of meal patterns. This review identified thirtyfour and fourteen studies that have examined associations between adults' meals patterns, nutrient intakes and diet quality, respectively. Most studies defined meals using a participant-identified approach, but varied in the additional criteria used to determine individual meals, snacks and/or eating occasions. Studies also varied in the types of meal patterns, nutrients and diet quality indicators examined. The most consistent finding was an inverse association between skipping breakfast and diet quality. No consistent association was found for other meal patterns, and little research has examined how meal timing is associated with diet quality. In conclusion, an understanding of the influence of different meal definitions on the characterisation of meal patterns will facilitate the interpretation of the existing literature, and may provide guidance on the most appropriate definitions to use.

Key words: Meal patterns: Diet quality: Nutrient intake: Diet quality indicators

\section{Introduction}

It is widely recognised that a nutritionally sound diet is fundamental to human health and wellbeing across the lifespan ${ }^{(1)}$. A poor diet contributes to poor health and is a well-established, modifiable risk factor for the development of non-communicable diseases, which are leading causes of death globally ${ }^{(1)}$. Traditionally, research has focused on the relationship between individual nutrients and health outcomes, yet this approach has often resulted in conflicting findings ${ }^{(2)}$. Hence there has been a gradual shift in the past decade towards less reductionist approaches to examining diet-disease relationships (for example, dietary patterns analysis) that better capture the interaction of nutrients and bioactive compounds within the whole diet ${ }^{(2,3)}$. However, people consume combinations of foods as meals and snacks rather than as individual foods and nutrients. Understanding the nutritional composition of meals and the ways in which different meal patterns make an impact on diet quality might help to elucidate important diet-disease relationships.
Moreover, a meals-based approach could complement current dietary advice, which currently uses a food-based framework (for example, the Australian Dietary Guidelines) ${ }^{(4)}$ to assist populations in achieving the recommended daily intakes of foods and nutrients. That is, dietary advice in the context of meals could help populations with their daily meal preparation and therefore be a more practical and salient way to assist populations to follow dietary guidelines.

Most of the research in this area, however, has focused on how different meal patterns (also referred to as eating patterns) make an impact on energy balance and weight status $^{(5,6)}$. An oft-cited drawback to interpreting the evidence from these studies has been the different approaches employed to operationally define meals and/ or snacks ${ }^{(5,7)}$. However, previous reviews of the impact of different definitions on the interpretation of meal pattern studies have examined snacking only ${ }^{(7,8)}$. Moreover, to date there has been no comprehensive review of studies investigating associations between meal patterns and diet

Abbreviations: AHEI, Alternative Healthy Eating Index; EI, energy intake; EO, eating occasion; FBC, food-based classification; HEI, Healthy Eating Index.

*Corresponding author: Rebecca M. Leech, fax +61392446017, email rleec@deakin.edu.au 
quality; previous reviews have focused on dietary contributions in relation to eating frequency or snacking ${ }^{(8,9)}$.

Therefore, the primary purpose of the present paper is to provide an overview and critique of meal pattern research, including previous approaches to the characterisation, definition and measurement of 'meals'. Second, the potential implication of these approaches will be further examined in a critical review of the literature of the contributions of meal patterns to energy and nutrient intakes and overall diet quality among adults.

\section{Characterisation of meals}

The term 'meal patterns' is an overarching construct that is often used to describe individuals' eating patterns at the level of a 'meal', such as a main meal (for example, breakfast, lunch or dinner) or a smaller-sized meal (for example, supper or snack). The neutral terms 'eating occasion' (EO) or 'eating event' are also used to describe any occasion where food or drink is ingested, and therefore incorporates all meal types. Meals have been described according to three constructs: (1) patterning (for example, frequency, spacing, regularity, skipping, timing); (2) format (for example, types of food combinations, sequencing of foods, nutrient profile/content); and (3) context (for example, eating with others or with the family, eating in front of the television or out of the home) (Table 1$)^{(10-13)}$. Table $1^{(14-34)}$ provides an overview of these constructs, including a description of the different meal patterns variables that have been examined previously along with their corresponding operational definitions. Examples of how meals have been measured in past studies are also presented in Table 1.

\section{Meal definitions}

To date, a variety of approaches has been used in the literature to define EO (meals and snacks). The approaches are summarised below and in Table $2^{(11,16,34-42)}$. The main approaches to defining meals are: participant-identified, time-of-day, food-based classification (FBC) and neutral. These definitions, along with examples from the literature and their respective advantages and disadvantages, are discussed below.

\section{Time-of-day}

As the name implies, this approach defines meals according to the time-of-day in which food was consumed. Explicitly, a 'meal' may be defined as the largest EO occurring between $06.00-10.00,12.00-15.00$ and $18.00-21.00$ hours, with smaller EO and EO falling outside of these times considered

Table 1. Overview of the three meal pattern constructs, and examples of variables currently assessed in the literature and the assessment methods that have been used to collect the meal pattern data

\begin{tabular}{|c|c|c|c|}
\hline Construct & Variable & Operational definition(s) & Example(s) of methods \\
\hline \multirow[t]{5}{*}{ Patterning } & $\begin{array}{l}\text { Frequency of EO } \\
\text { (meals and snacks) }\end{array}$ & Mean number of EO/meals/snacks per $\mathrm{d}^{(14)}$ & $\begin{array}{l}\text { Dietary recall }(24 \mathrm{~h})^{(14)} \text { Weekly } \\
\text { food diary }{ }^{(15)} \text { Meal patterns } \\
\text { questionnaire }^{(16)} \text { Single questionnaire } \\
\text { items }^{(17)}\end{array}$ \\
\hline & Spacing of EO & Mean time between $\mathrm{EO}^{(14)}$ & Dietary recall $(24 \mathrm{~h})^{(14)}$ \\
\hline & Regularity of meals & $\begin{array}{l}\text { Consistency of EO frequency and spacing }{ }^{(18)} \\
\text { Usually eats breakfast, lunch and dinner } \\
\text { each day }{ }^{(19-21)}\end{array}$ & $\begin{array}{l}\text { Semi-quantitative food records }{ }^{(18)} \\
\text { Single questionnaire items } \\
\text { Food records }(3 \mathrm{~d})^{(21)}\end{array}$ \\
\hline & Meal skipping & Usually omits breakfast, lunch or dinner ${ }^{(17,22)}$ & $\begin{array}{l}\text { Single questionnaire items }{ }^{(17)} \text { Meal } \\
\text { patterns questionnaire }{ }^{(22)} \text { Dietary } \\
\text { recall }(24 \mathrm{~h})^{(23)}\end{array}$ \\
\hline & Meal timing & $\begin{array}{l}\text { The timing of breakfast, lunch or dinner (early/late) } \\
\text { Time-of-day wherein majority of daily El is consumed } \\
\text { (morning, midday, evening/late) } \\
\text { (eating after going to bed) } \\
(28)\end{array}$ & $\begin{array}{l}\text { Food records }(7 \mathrm{~d})^{(24)} \text { Prescribed diet } \\
\quad \text { (intervention studies) })^{(26)} \text { Dietary } \\
\text { recall }(24 \mathrm{~h})^{(27)} \text { Single questionnaire } \\
\text { items }^{(28)}\end{array}$ \\
\hline \multirow[t]{3}{*}{ Format } & $\begin{array}{l}\text { Meal food } \\
\text { type/combinations }\end{array}$ & Classifications of combinations of foods in meals ${ }^{(29)}$ & Food records $(7 \mathrm{~d})^{(29)}$ \\
\hline & Meal food sequencing & $\begin{array}{l}\text { Temporal distribution of consumption of food groups and } \\
\text { intake of energy and nutrients within a meal }\end{array}$ & Food records $(2 \mathrm{~d})^{(30)}$ \\
\hline & Nutrient composition & $\begin{array}{l}\text { Energy, protein, fat and carbohydrate composition } \\
\text { of a meal }\end{array}$ & $\begin{array}{l}\text { Prescribed diet (intervention studies) })^{(26)} \\
\text { Weekly food diary }{ }^{(31)}\end{array}$ \\
\hline \multirow[t]{3}{*}{ Context } & $\begin{array}{l}\text { Presence of others at } \\
\text { a meal (for example, } \\
\text { friends/family) }\end{array}$ & $\begin{array}{l}\text { Types of food eaten in different social contexts } \\
\text { (for example, alone } v \text {. with others) }{ }^{\left({ }^{(32)}\right.} \text { Macronutrient } \\
\text { composition of meals eaten alone } v \text {. with others }{ }^{(33)}\end{array}$ & $\begin{array}{l}\text { Personal digital assistants }{ }^{(32)} \\
\text { Weekly food diary }\end{array}$ \\
\hline & $\begin{array}{l}\text { Eating while doing activities } \\
\text { (for example, watching TV) }\end{array}$ & $\begin{array}{l}\text { Types of food consumed while watching TV } \\
v \text {. other activities } \\
\text { (32,34) }^{-1}\end{array}$ & $\begin{array}{l}\text { Personal digital assistants }{ }^{(32)} \text { Food } \\
\text { records and ecological momentary } \\
\text { assessment }^{(34)}\end{array}$ \\
\hline & $\begin{array}{l}\text { Meal location (for example, } \\
\text { eating at home } v \text {. eating out) }\end{array}$ & $\begin{array}{l}\text { Energy and macronutrient content of meals consumed } \\
\text { at home } v \text {. out of the home } e^{(30)} \text { Types of food } \\
\text { consumed by location }{ }^{(32)}\end{array}$ & $\begin{array}{l}\text { Food records }(2 \mathrm{~d})^{(30)} \text { Personal } \\
\quad \text { digital assistants }^{(32)}\end{array}$ \\
\hline
\end{tabular}

EO, eating occasions; El, energy intake; TV, television. 


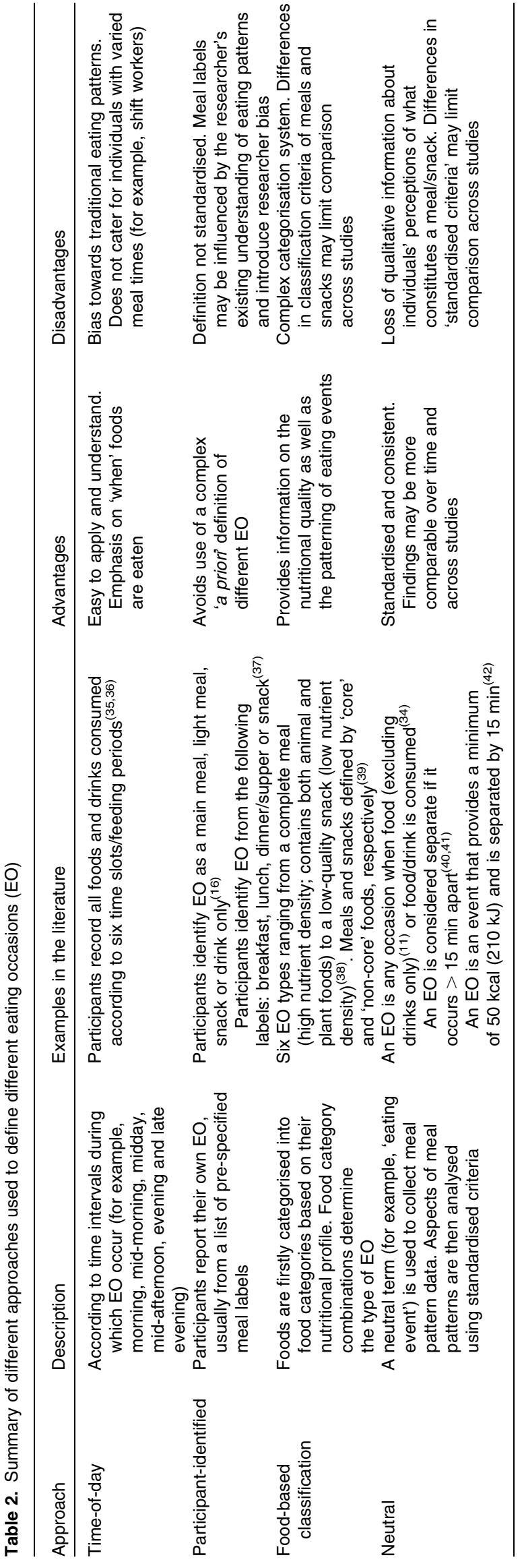

as snacks ${ }^{(43)}$. While this approach is easy to understand and apply, the time parameters used are not always explicit, the number of meals per $\mathrm{d}$ is usually restricted to a maximum of three and it does not capture meals eaten at unusual times, such as among shift workers ${ }^{(35,36)}$. Ultimately, a time-ofday definition requires a measure of time of eating. It is also subject to bias of the researcher, as the time intervals to define a meal or snack are often based on their understanding of eating patterns, potentially influenced by local or cultural factors ${ }^{(44)}$

\section{Participant-identified}

This definition relies on the respondent to identify an EO as a meal or snack, often from a list of pre-specified meal labels (for example, breakfast, lunch, dinner/supper or snack $)^{(44)}$. While this definition avoids the imposition of a complex criterion to classify EO as meals or snacks ${ }^{(45-47)}$, it is not standardised due to subjectivity in participants' allocation of an eating event as a meal or snack $^{(44,48)}$. Chamontin et al. ${ }^{(49)}$ showed that the word 'snack', when used in its verb form (for example, 'When did you last snack?'), elicited different conceptual responses from participants than when snack was used as a noun (for example, "When did you last have a snack?'). However, not all studies ask respondents to identify the $\mathrm{EO}^{(43)}$.

\section{Food-based classification}

Lennernäs \& Andersson ${ }^{(38)}$ developed the concept of a FBC of $\mathrm{EO}$ intended to reflect both qualitative and quantitative aspects of meal patterns. Initially, foods consumed were sorted into seven food categories that differed by nutritional profile (for example, animal/plant origin, nutrient density, energy density) and second, depending on the combination of food categories consumed, eating events were classified as one of six types of EO ranging from a 'complete meal' to a 'low-quality snack'. Another variation of the FBC system, based on 'core' and 'non-core' foods has since been developed ${ }^{(39)}$, but generally the FBC of EO has had limited uptake, probably due to the complexity of the FBC criteria. While this definition of a meal can capture the types of foods eaten, the researcher must decide which criteria should be used to classify meals and snacks (for example, a criterion based on different nutrient profiles $v$. a criterion based on the energy density of foods).

\section{Neutral}

In 1999, Mäkelä et al. ${ }^{(11)}$ recognised that conventional meal labels are culturally laden and therefore may mean different things for people from different cultural backgrounds. This led the authors to use the neutral term 'eating event' for an occasion where food was consumed. Once empirical data had been collected, different dimensions of meal 
patterns using standardised criteria (for example, time-ofday, number of hot/cold eating events) were used to describe the data. The advantage of a neutral definition is that it can be standardised and can allow for comparison across different population groups and cultures. However, despite this neutral definition, additional criteria have been applied in the literature with regards to the time intervals between $\mathrm{EO}$, a minimum-energy criterion to define each individual EO and whether beverage-only EO are included or excluded. It is important to note that these additional criteria have also been applied to the time-of-day and participant-identified definitions in order to define an 'individual' or 'separate' meal and/or snack ${ }^{(15,50,51)}$, thus adding another layer of diversity to these meal definitions.

When the time of eating is recorded, researchers must decide how to delineate separate $\mathrm{EO}^{(35,52)}$. Indeed, the period of time used to separate different $\mathrm{EO}$ varies across studies, with intervals of $15 \mathrm{~min}^{(40,42,50)}, 30 \mathrm{~min}^{(53)}$ or $45 \min ^{(15)}$ or $1 \mathrm{~h}^{(51)}$ reported. Some studies also include a minimum energy criterion as part of the meal definition. For example, in some studies, EO were only treated as an EO if they contributed a minimum amount of energy (for example, $210 \mathrm{~kJ})^{(15,42,54)}$. These variations in criteria are likely to make an impact on the frequency, spacing and nutritional contribution of the EO reported and on associations with health outcomes. In support of this, Murakami \& Livingstone ${ }^{(55)}$ found the number of reported EO per d was reduced by two or more EO for both men and women after applying a minimum-energy criterion of $210 \mathrm{~kJ}$. In the same study, the different definitions of an EO greatly affected the results of the association between eating frequency and BMI and waist circumference.

The methodological differences in spacing between EO and energy content may also indirectly influence the inclusion $^{(50)}$ or exclusion ${ }^{(56)}$ of beverage-only occasions as part of the meal definition. A larger time interval criterion applied to $24 \mathrm{~h}$ recall data to separate individual EO may not be able to capture smaller EO (including beverage-only occasions). The findings from one study suggest that smaller intervals may also be useful to detect important changes in energy intake (EI) from beverageonly occasions over time ${ }^{(14)}$.

\section{Measurement of meals}

There have been a number of different approaches to the measurement of meals. Much data on meal patterns have been derived from dietary assessment methods such as $24 \mathrm{~h}$ recalls and food records. These methods provide detailed information on the types and quantities of food/ beverages consumed and, usually, time of consumption $^{(14,15,36,50,57)}$

During a $24 \mathrm{~h}$ recall, participants may be asked to report the type of $\mathrm{EO}$ as a main meal or a snack ${ }^{(14,50,57)}$, whereas food records are often segregated by the researcher into meal time slots (for example, pre-breakfast, breakfast, mid-morning, etc. $)^{(35,36)}$. Contextual information is not always collected as part of the recall or food record and thus only examination of meal patterning may be possible. While meal format could be examined with this type of data, little research in this area has been conducted. One possible reason for this is that there has been little exploration of statistical techniques that are able to examine complex combinations and sequencing of foods at a meal. Hearty \& Gibney ${ }^{(29)}$ explored the potential use of supervised data-mining techniques in meal pattern analysis, specifically to predict diet quality based on different combinations of foods at a meal. However, to our knowledge, this is the only study that has applied these analytic tools in meal pattern analysis.

Many food diaries collect data on time of eating, and/or self-identified meals, and similar to $24 \mathrm{~h}$ recalls, some collect contextual information (for example, location of eating, presence of others). The weekly food diary method developed by de Castro ${ }^{(58)}$, in addition to the time and amount of food eaten over a $7 \mathrm{~d}$ period, asks participants to record detailed contextual information (for example, mood and hunger levels before eating, the number and nature of other people eating with them). While this method elicits rich contextual information, participant burden is high, thus reducing its practicality in larger-scale studies. In a recent study, participants used personal digital assistant devices to record real-time information on dietary intake, EO type, location and context ${ }^{(32)}$. As a result, the researchers were able to assess contextual influences on the types of foods that participants consumed at an EO. However, while this type of assessment method lends itself to the examination of meal patterning, format and context, these 'real-time' assessment devices have not yet been extensively developed ${ }^{(59)}$.

FFQ are also commonly used to collect dietary data, particularly in large-scale studies ${ }^{(60)}$. While FFQ provide estimates of the frequency and types of foods that are usually consumed, they do not provide data that directly allow examination of $\mathrm{EO}$, and additional questionnaires ${ }^{(16,56)}$ or short questionnaire items ${ }^{(17)}$ have been used to collect information on meal patterns. Example of questionnaire items include: 'Indicate the times of day you usually eat ${ }^{\text {(17) }}$, 'Do you eat regular breakfast, lunch and dinner or evening meal each day? ${ }^{\text {(19) }}$ or 'Do you usually have the following meals (breakfast, lunch, snack, dinner, evening snack)? ${ }^{(20)}$. Some questionnaires may only ask about 'eating' frequency, and thus may not capture beverage-only EO. The reliability and validity of meal pattern questionnaires are often not reported ${ }^{(16)}$

\section{Associations between meal patterns, nutrient intakes and overall diet quality}

Due to the current limited methods available to collect meal pattern data, most research to date has examined meal patterning ${ }^{(5,61)}$, with relatively little focus on meal 
format ${ }^{(29)}$ and context ${ }^{(32)}$. As stated previously, studies on meal patterning have examined meal frequency, spacing, skipping and timing. However, these studies have differed in their approach to defining meals, and even within a given approach, there have been differences in the delineation of individual EO, meals and/or snacks. The ways these different methodological differences affect the characterisation of meal patterns have been little explored $^{(55)}$ and, to the best of our knowledge, how these differences affect the associations between meal patterns and nutrient intakes or diet quality has not previously been examined. Understanding the relationships between adults' meal patterns and nutrient intakes and diet quality is necessary to determine if they are markers of the healthiness and variety of the whole diet ${ }^{(62)}$. Therefore, the associations between 'meal patterning', nutrient intake and overall diet quality among adults were examined considering the impact of different meal definitions used for the characterisation of meal patterns.

A literature search was undertaken in the PubMed and EMBASE electronic databases using the following terms: meal, snack, breakfast, lunch, dinner, eating frequency, eating pattern, eating behavior, eating behaviour, eating occasion, eating episode, diet quality, dietary quality, dietary pattern, dietary behavior, dietary behaviour, nutritional quality, dietary intake, food intake, energy intake, nutrient, macronutrient, dietary composition and nutritional composition. The search terms were limited to the title/abstract and the following filters were applied: journal article, humans, adult and English. Two searches were conducted; the first between February and May 2013 and the second between February and April 2014. The criteria for inclusion in the review were: original research studies that examined the nutritional contributions of meal patterns or associations between meal patterns and nutrient intakes and/or overall diet quality in free-living, healthy men and women aged 19 years and over. Diet quality was defined as the quality of a individual's overall food intake determined by compliance with national dietary guidelines or an a priori diet quality score ${ }^{(63)}$. Studies that examined populations with conditions or circumstances that may affect meal patterns (for example, elite athletes, shiftworkers, individuals with chronic diseases, recipients of meal programmes and pregnant or breast-feeding women), or examined associations with EI only, were excluded.

\section{Characteristics of studies which examined associations between meal patterns and nutrient intakes}

A total of thirty-four studies (Table 3) were identified which examined the nutritional contribution of different meal patterns, in adults. However, only thirteen of these examined more than one micronutrient ${ }^{(23,30,46,47,52,64-71)}$. All except two studies ${ }^{(35,72)}$ were cross-sectional. Of the studies, fifteen and five studies were conducted in the
$\mathrm{USA}^{(41,46,48,52,57,68,70,72-79)}$ and Scandinavia $^{(45,47,53,65,80)}$, respectively, with fewer studies conducted in Western Europe ${ }^{(15,30,67,81)}$, the $\mathrm{UK}^{(35,40,82)}$, East Asia ${ }^{(64,71,83)}$, Australia $^{(36,69)}$, Canada $^{(66)}$ and Brazil ${ }^{(84)}$. Meal patterns were mostly participant-identified ${ }^{(15,23,45-48,52,53,57,66,69,70,75,77,78,82)}$, although these studies varied in the additional criteria used to determine an individual EO, meal and/or snack, and their treatment of beverages. For example, eleven studies $^{(23,45-47,52,66,69,75,78,80,82)}$ applied no additional criteria, whereas in other studies, EO were delineated using 15 -min time intervals ${ }^{(57,70,77)}$, a 30-min interval ${ }^{(53)}$, a 45-min interval plus a $50 \mathrm{kcal}(210 \mathrm{~kJ})$ energy criterion ${ }^{(15)}$ and a 59-min interval (applied to meals only) ${ }^{(48)}$. All beverage types (energy and non-energy) could constitute an individual EO in nine studies ${ }^{(35,45-47,53,66,70,75,77)}$, whereas other studies excluded water beverages ${ }^{(23,52)}$, non-nutritive beverages (for example, water, tea, black coffee $)^{(80)}$ or did not address/include beverages as part of the definition ${ }^{(48,57,71,78,82)}$. Time-of-day definitions were also common ${ }^{(35,36,64,67,72,76)}$ as well as a combination of two definitions (for example, self-identified and timeof-day, or time-of-day and type/combination of foods eaten) ${ }^{(30,65,68,73,81)}$.

The most common methods used to assess both dietary intake and meal patterns were $24 \mathrm{~h}$ recalls $^{(23,46,48,52,57,66,68-70,73,76,77,83)}$ or food records $(2-7 \text { d })^{(15,30,35,36,40,41,57,64,65,79,81)}$. Only four studies excluded energy misreporters ${ }^{(48,80)}$ or energy under-reporters $^{(40,41)}$. There was significant variation in the aspects of meal patterning examined and these aspects could be broadly categorised as: meals $v$. snacks, eating frequency, meal skipping/regularity and meal timing. These categories are used below to direct discussion on the studies' findings in relation to associations with nutrient intakes. The potential impact of different definitions on the characterisation of meal patterns and their associations with nutrient intakes is also discussed.

\section{Meals $v$. snacks in relation to nutrient intakes}

A total of ten studies ${ }^{(15,30,35,36,65,73,75,79,81,84)}$ were identified that examined the contributions of meals and/or snacks to energy and nutrient intakes. In a prospective study of 1253 adults from the UK, Almoosawi et al. ${ }^{(35)}$ examined 17-year changes in the contributions of breakfast, lunch and dinner to macronutrient intake. The authors found that the lunch and evening meal contributed the greatest proportion of total daily energy, protein, fat and carbohydrate intake, which was consistent over time. This is supported by other research highlighting that main meals are when the largest volume of food is normally consumed ${ }^{(15,81)}$. When the nutritional contribution of meals and snacks are analysed relative to their contribution to EI, a finding across five studies was that snacks provided a lower proportion of total energy from fat and/or protein than did meals $^{(15,36,48,65,73)}$. This finding was consistent despite the 


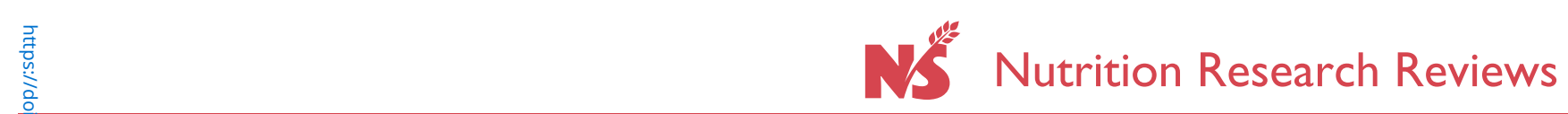

Table 3. Continued

\begin{tabular}{|c|c|c|c|c|c|c|c|c|c|}
\hline First author (year) & $\begin{array}{l}\text { Country } \\
\text { and study } \\
\text { design }\end{array}$ & Sample & $\begin{array}{l}\text { Aspect(s) of meal } \\
\text { patterns } \\
\text { examined }\end{array}$ & $\begin{array}{l}\text { Diet and meal } \\
\text { pattern } \\
\text { measure }\end{array}$ & $\begin{array}{l}\text { Meal or snack } \\
\text { definition }\end{array}$ & Macronutrients & $\begin{array}{l}\text { Other dietary } \\
\text { components }\end{array}$ & Covariates & Selected key findings \\
\hline Coates $(2002)^{(72)}$ & $\begin{array}{l}\text { USA } \\
\text { Case-control }\end{array}$ & $\begin{array}{l}2380 \text { healthy control } \\
\text { men and women, } \\
30-79 \text { years }\end{array}$ & Eating frequency & $\begin{array}{l}\text { Dietary history } \\
\text { interview }\end{array}$ & Time-of-day & & $\mathrm{El}, \mathrm{Ca}$, fibre & & $\begin{array}{l}\text { Control men and women eating } \\
1-2 \text { times/d were more likely } \\
\text { to have lower intakes of } \\
\text { energy, Ca and fibre than } \\
\text { those eating } \geq 3 \text { times/d } \\
(P<0.001)\end{array}$ \\
\hline Dattilo (2011) ${ }^{(84)}$ & Brazil & $\begin{array}{l}24 \text { men and } 28 \\
\text { women, } 19-45 \\
\text { years }\end{array}$ & $\begin{array}{l}\text { Meal distribution } \\
\text { across the day/ } \\
\text { meal timing }\end{array}$ & $\begin{array}{l}\text { Dietary recall } \\
\text { (recall period } \\
\text { not given) }\end{array}$ & Not clear & Protein, fat, $\mathrm{CHO}$ & El & Stratified by sex & $\begin{array}{l}\text { Among women only, El was } \\
\text { significantly higher }(P<0 \cdot 01) \\
\text { at lunch than at breakfast, } \\
\text { snacks and at supper. CHO } \\
\text { and protein intake at night } \\
\text { (among men) and afternoon } \\
\text { (among women) was higher } \\
\text { in than in the morning. A } \\
\text { higher proportion of daily EI } \\
\text { in the afternoon and evening } \\
\text { was associated with lower } \\
\text { and higher overall El, } \\
\text { respectively }(P<0.05)\end{array}$ \\
\hline de Castro $(2004)^{(79)}$ & $\begin{array}{l}\text { USA } \\
\text { C/S }\end{array}$ & $\begin{array}{l}375 \text { men and } 492 \\
\text { women, mean age } \\
36.3(\text { SD 13.8) } \\
\text { years }\end{array}$ & $\begin{array}{l}\text { Meal distribution } \\
\text { across the day/ } \\
\text { meal timing }\end{array}$ & $7 \mathrm{dFR}$ & Neutral $^{\star}$ & Protein, fat, $\mathrm{CHO}$ & El & $\begin{array}{l}\text { Stratified by sex. } \\
\text { Sensitivity anal- } \\
\text { ysis excluding } \\
\text { energy under- } \\
\text { reporters }\end{array}$ & $\begin{array}{l}\text { Intakes per meal of energy as } \\
\text { CHO, fat, protein and alcohol } \\
\text { were significantly higher in the } \\
\text { evening period }(18.00-22.00 \\
\text { hours) than the other four } \\
\text { periods of the day }(P<0.05) \text {. } \\
\text { Among both sexes, a higher } \\
\text { proportion of daily El in the } \\
\text { morning and evening was } \\
\text { associated with lower and } \\
\text { higher overall El, respectively } \\
(P<0.01)\end{array}$ \\
\hline $\begin{array}{l}\text { Deshmukh-Taskar } \\
(2010)^{(23)}\end{array}$ & $\begin{array}{l}\text { USA } \\
\text { C/S }\end{array}$ & $\begin{array}{l}2615 \text { men and } \\
\text { women, 20-39 } \\
\text { years }\end{array}$ & Breakfast skipping & $24 \mathrm{HR}$ & $\begin{array}{l}\text { Participant- } \\
\text { identified }^{\star}\end{array}$ & $\begin{array}{l}\text { Protein, fat, CHO, } \\
\text { added and } \\
\text { total sugars, } \\
\text { SFA, MUFA, } \\
\text { PUFA, discre- } \\
\text { tionary oils and } \\
\text { solid fats }\end{array}$ & $\begin{array}{l}\text { El, fibre, vitamins } \\
\mathrm{A}, \mathrm{B}_{6}, \mathrm{~B}_{12}, \mathrm{C} \\
\mathrm{D} \text { and } \mathrm{E}, \text { thia- } \\
\text { min, riboflavin, } \\
\text { niacin, folate, } \\
\mathrm{Zn}, \mathrm{Ca}, \mathrm{Fe}, \\
\mathrm{Mg}, \mathrm{P}, \mathrm{Na}, \mathrm{K} \\
\text { and choles- } \\
\text { terol }\end{array}$ & $\begin{array}{l}\text { El, age, ethnicity, } \\
\text { sex, sex } \times \\
\text { ethnicity, poverty } \\
\text { income ratio, } \\
\text { smoking, PA, } \\
\text { marital status } \\
\text { and alcohol } \\
\text { intake }\end{array}$ & $\begin{array}{l}\text { Compared with breakfast consu- } \\
\text { mers, total energy, dietary } \\
\text { fibre, vitamin } \mathrm{A} \text {, thiamin, } \\
\text { riboflavin, vitamin } \mathrm{B}_{12} \text {, folate, } \\
\mathrm{Ca}, \mathrm{P}, \mathrm{Mg} \text { and } \mathrm{K} \text { intakes were } \\
\text { significantly lower in breakfast } \\
\text { skippers }(P<0.0001) \text {. Nutrient } \\
\text { adequacy ratios for vitamins } \\
\mathrm{A} \text { and } \mathrm{C}, \mathrm{Ca}, \mathrm{Mg}, \mathrm{K} \text { and fibre } \\
\text { were also significantly lower } \\
\text { among breakfast skippers } \\
(P<0.01)\end{array}$ \\
\hline Drummond $(1998)^{(40)}$ & $\begin{array}{l}\text { Scotland } \\
\mathrm{C} / \mathrm{S}\end{array}$ & $\begin{array}{l}48 \text { men and } 47 \\
\text { women, } 20-55 \\
\text { years }\end{array}$ & Eating frequency & $7 \mathrm{~d} \mathrm{FR \dagger}$ & Neutral¥ & $\begin{array}{l}\text { Protein, } \mathrm{CHO} \text {, fat, } \\
\text { sugar }\end{array}$ & El, alcohol & Stratified by sex & $\begin{array}{l}\text { Eating frequency was positively } \\
\text { correlated with total EI }(r 0.31 \text {, } \\
P=0.01 \text { ) among women only } \\
\text { and percentage of energy } \\
\text { from CHO (women: } r 0.38, \\
P=0.02 \text { and men: } r 0.3 \text {, } \\
P=0.05 \text { ) }\end{array}$ \\
\hline Duval $(2008)^{(41)}$ & $\begin{array}{l}\text { USA } \\
\text { C/S }\end{array}$ & $\begin{array}{l}85 \text { women, } 47-56 \\
\text { years }\end{array}$ & Eating frequency & $7 \mathrm{~d} F \mathrm{~F} \dagger$ & Neutral & Protein, $\mathrm{CHO}$, fat & El, alcohol & & $\begin{array}{l}\text { Eating frequency was positively } \\
\text { correlated with total EI }(r 0.41 \text {, } \\
P=0.005) \text { and percentage of } \\
\text { energy from } \mathrm{CHO}(r 0.21, \\
P=0.045) \text { and total weight of } \\
\mathrm{CHO}(r 0.37, P=0.001) \text { and } \\
\text { protein }(r 0.31, P=0.005)\end{array}$ \\
\hline
\end{tabular}




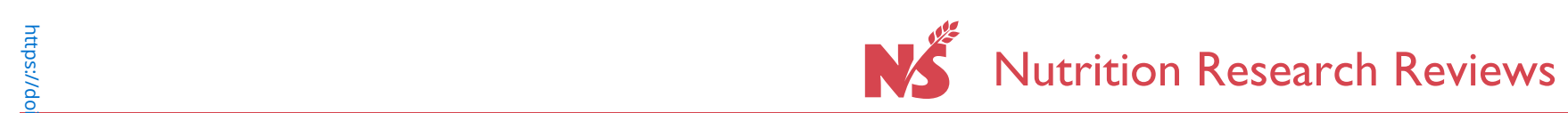

Table 3. Continued

\begin{tabular}{|c|c|c|c|c|c|c|c|c|c|}
\hline First author (year) & $\begin{array}{l}\text { Country } \\
\text { and study } \\
\text { design }\end{array}$ & Sample & $\begin{array}{l}\text { Aspect(s) of meal } \\
\text { patterns } \\
\text { examined }\end{array}$ & $\begin{array}{l}\text { Diet and meal } \\
\text { pattern } \\
\text { measure }\end{array}$ & $\begin{array}{l}\text { Meal or snack } \\
\text { definition }\end{array}$ & Macronutrients & $\begin{array}{l}\text { Other dietary } \\
\text { components }\end{array}$ & Covariates & Selected key findings \\
\hline Edelstein $(1992)^{(78)}$ & $\begin{array}{l}\text { USA } \\
\text { C/S }\end{array}$ & $\begin{array}{l}2034 \text { white adults, } \\
50-89 \text { years }\end{array}$ & Eating frequency & $\begin{array}{l}\text { FFQ } \\
\text { Meal patterns: } \\
\text { one } Q \text { item }\end{array}$ & $\begin{array}{l}\text { Participant- } \\
\text { defined }\end{array}$ & Fat, SFA & $\begin{array}{l}\text { El, cholesterol, } \\
\text { fibre }\end{array}$ & Age and sex & $\begin{array}{l}\text { Eating frequency was positively } \\
\text { associated with EI, total fat } \\
\text { and SFA }(P<0.001) \text { and total } \\
\text { fibre }(P=0.012)\end{array}$ \\
\hline Hampl (2003) ${ }^{(46)}$ & $\begin{array}{l}\text { USA } \\
\text { C/S }\end{array}$ & $\begin{array}{l}1756 \text { men and } 1511 \\
\text { women, } 39-43 \\
\text { years }\end{array}$ & $\begin{array}{l}\text { Snacking frequency } \\
\text { and timing }\end{array}$ & $2 \times 24 \mathrm{HR}$ & $\begin{array}{l}\text { Participant- } \\
\text { identified }{ }^{\star}\end{array}$ & $\mathrm{CHO}$, fat, protein & $\begin{array}{l}\text { El, all nutrients } \\
\text { with an RDA } \\
\text { except vita- } \\
\text { mins D and K, } \\
\text { Se and I }\end{array}$ & $\begin{array}{l}\text { El stratified by sex } \\
\text { and 'snacker' } \\
\text { type }\end{array}$ & $\begin{array}{l}\text { Male and female multiple snack- } \\
\text { ers had lower intakes of pro- } \\
\text { tein, cholesterol and Na, but } \\
\text { higher El and Ca intake com- } \\
\text { pared with non-snackers. } \\
\text { Female evening snackers had } \\
\text { significantly higher El than did } \\
\text { morning snackers }\end{array}$ \\
\hline Holmbäck $(2010)^{\left({ }^{(8)}\right)}$ & $\begin{array}{c}\text { Sweden } \\
\text { C/S }\end{array}$ & $\begin{array}{l}1355 \text { men and } 1654 \\
\text { women, } 47-68 \\
\text { years }\end{array}$ & Eating frequency & $\begin{array}{l}\text { Diet history } \\
\text { interview } \dagger \\
\text { Meal patterns: } \\
\text { Q }\end{array}$ & $\begin{array}{l}\text { Participant- } \\
\text { identified }\end{array}$ & $\mathrm{CHO}$, fat, protein & $\begin{array}{l}\text { El, fibre, } \mathrm{Fe}, \\
\mathrm{Ca}, \mathrm{Mg}, \\
\beta \text {-carotene, } \\
\text { ascorbic acid, } \\
\text { vitamin } \mathrm{E}, \\
\text { folate and } \\
\text { alcohol }\end{array}$ & $\begin{array}{l}\text { Stratified by sex } \\
\text { and excluded } \\
\text { individuals with } \\
\text { past food habit } \\
\text { change }\end{array}$ & $\begin{array}{l}\text { Total El and percentage energy } \\
\text { from CHO significantly } \\
\text { increased with increased } \\
\text { eating frequency }(P<0.01) \text {. } \\
\text { Percentage energy from fat } \\
\text { (women only), protein and } \\
\text { fibre density decreased. Nutri- } \\
\text { ent densities of vitamin C, } \\
\text { folate and Fe were signifi- } \\
\text { cantly higher among women } \\
\text { who ate } \geq 6 \text { times/d } \\
(P<0.01)\end{array}$ \\
\hline Howarth $(2007)^{(48)}$ & $\begin{array}{l}\text { USA } \\
\text { C/S }\end{array}$ & $\begin{array}{l}1792 \text { young adults, } \\
20-59 \text { years; and } \\
893 \text { older adults, } \\
60-90 \text { years }\end{array}$ & $\begin{array}{l}\text { Meal and snack } \\
\text { frequency, meal } \\
\text { skipping }\end{array}$ & $2 \times 24 \mathrm{HR} \dagger$ & $\begin{array}{l}\text { Participant- } \\
\text { identified }\end{array}$ & $\mathrm{CHO}$, fat, protein & $\begin{array}{l}\text { El, fibre, fibre } \\
\text { density }\end{array}$ & Age group & $\begin{array}{l}\text { Among both age groups lunch } \\
\text { and dinner provided the } \\
\text { highest proportion of El from } \\
\text { protein and fat and snacks } \\
\text { provided the least amount of } \\
\text { fibre density } \\
\text { Dinner provided the highest } \\
\text { fibre density among younger } \\
\text { adults. Among older adults, } \\
\text { breakfast was higher in fibre } \\
\text { density }\end{array}$ \\
\hline Kant $(1997)^{(76)}$ & $\begin{array}{l}\text { USA } \\
\text { Prospective } \\
\text { (mean } \\
\text { follow-up } \\
10.1 \text { years) }\end{array}$ & $\begin{array}{l}2580 \text { men and } 4567 \\
\text { women, 25-74 } \\
\text { years }\end{array}$ & $\begin{array}{l}\text { Meal timing } \\
\quad \text { (evening eating) }\end{array}$ & $24 \mathrm{HR}$ & $\begin{array}{l}\text { Time-of-day } \\
\text { (eating after } \\
17.00 \text { hours) }\end{array}$ & $\mathrm{CHO}$, fat, protein & $\mathrm{El}$, alcohol & $\begin{array}{l}\text { Stratified by sex, } \\
\text { age, and El } \\
\text { (unless EI was } \\
\text { the dependent } \\
\text { variable) }\end{array}$ & $\begin{array}{l}\mathrm{C} / \mathrm{S} \text { analysis of baseline data } \\
\text { showed that with increasing } \\
\text { percentage of energy } \\
\text { consumed after } 17.00 \text { hours, } \\
\text { mean daily energy and alcohol } \\
\text { intake increased and percen- } \\
\text { tage energy from CHO } \\
\text { decreased }\end{array}$ \\
\hline Kearney $(2001)^{(30)}$ & $\begin{array}{l}\text { Holland } \\
\text { C/S }\end{array}$ & $\begin{array}{l}\text { About } 6000 \text { partici- } \\
\text { pants (age/sex not } \\
\text { provided) }\end{array}$ & $\begin{array}{l}\text { Contribution of } \\
\text { meals } v \text {. snacks to } \\
\text { nutrient intakes }\end{array}$ & $2 d F R$ & $\begin{array}{l}\text { Time-of-day and } \\
\text { type of foods } \\
\text { eaten (for } \\
\text { example, } \\
\text { lunch }= \\
\text { bread meal; } \\
\text { dinner = hot } \\
\text { meal) }\end{array}$ & $\begin{array}{l}\text { Protein, animal } \\
\text { and vegetable } \\
\text { protein, fat, } \\
\text { SFA, MUFA, } \\
\text { PUFA, CHO }\end{array}$ & $\begin{array}{l}\text { El, cholesterol, } \\
\text { fibre, Ca, } \mathrm{P}, \\
\mathrm{Fe} \text {, haem } \mathrm{Fe}, \\
\text { non-haem } \mathrm{Fe}, \\
\mathrm{Zn} \text { and } \\
\text { vitamins } \mathrm{B}_{1}, \\
\mathrm{~B}_{2}, \mathrm{~B}_{6}, \mathrm{D}, \mathrm{E} \\
\text { and } \mathrm{C}\end{array}$ & - & $\begin{array}{l}\text { The (hot) dinner meal was the } \\
\text { main contributor to the intake } \\
\text { of all micronutrients except } \\
\text { Ca. The dinner meal also } \\
\text { provided } 71 \% \text { of haem Fe } \\
\text { and the lunch (bread) meal } \\
\text { was the main contributor of } \\
\text { Ca intakes }\end{array}$ \\
\hline
\end{tabular}




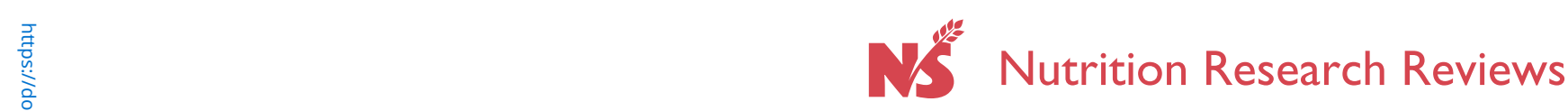

Table 3. Continued

\begin{tabular}{|c|c|c|c|c|c|c|c|c|c|}
\hline First author (year) & $\begin{array}{l}\text { Country } \\
\text { and study } \\
\text { design }\end{array}$ & Sample & $\begin{array}{l}\text { Aspect(s) of meal } \\
\text { patterns } \\
\text { examined }\end{array}$ & $\begin{array}{l}\text { Diet and meal } \\
\text { pattern } \\
\text { measure }\end{array}$ & $\begin{array}{l}\text { Meal or snack } \\
\text { definition }\end{array}$ & Macronutrients & $\begin{array}{l}\text { Other dietary } \\
\text { components }\end{array}$ & Covariates & Selected key findings \\
\hline Kerver $(2006)^{(52)}$ & $\begin{array}{l}\text { USA } \\
\text { C/S }\end{array}$ & $\begin{array}{l}15978 \text { adults, } \geq 20 \\
\text { years }\end{array}$ & $\begin{array}{l}\text { Eating frequency, } \\
\text { meal skipping }\end{array}$ & $24 \mathrm{HR}$ & $\begin{array}{l}\text { Participant- } \\
\text { identified }{ }^{*}\end{array}$ & Protein, $\mathrm{CHO}$, fat & $\begin{array}{l}\text { El, cholesterol, } \\
\text { vitamins } \mathrm{B}_{6} \\
\text { and } \mathrm{C} \text {, folic } \\
\text { acid, } \mathrm{Fe}, \mathrm{Ca} \text {, } \\
\mathrm{Mg}, \mathrm{Na}, \mathrm{K} \text {, } \\
\text { fibre }\end{array}$ & $\begin{array}{l}\text { Age group, sex, } \\
\text { ethnicity, income, } \\
\text { smoking status, } \\
\text { alcohol intake, } \\
\text { vitamin and min- } \\
\text { eral supplement } \\
\text { use, BMI, PA }\end{array}$ & $\begin{array}{l}\text { More frequent eaters had higher } \\
\text { intakes of } \mathrm{CHO} \text {, folic acid, } \\
\text { vitamin } \mathrm{C}, \mathrm{Ca}, \mathrm{Mg}, \mathrm{Fe}, \mathrm{K} \text { and } \\
\text { fibre and lower intakes of fat, } \\
\text { protein and cholesterol than } \\
\text { those who ate } 1-2 \text { times } / \mathrm{d} \\
\text { Breakfast skippers had the } \\
\text { lowest intake of all micro- } \\
\text { nutrients except } \mathrm{Na}\end{array}$ \\
\hline Khan $(1982)^{(75)}$ & $\begin{array}{l}\text { USA } \\
\text { C/S }\end{array}$ & $\begin{array}{l}71 \text { men and } 179 \\
\text { women students, } \\
\leq 25 \text { years }\end{array}$ & $\begin{array}{l}\text { Contribution of meals } \\
v . \text { snacks to energy } \\
\text { and nutrient intakes }\end{array}$ & $\begin{array}{l}\mathrm{Q} \text { (based on a } \\
24 \mathrm{HR} \text { ) }\end{array}$ & $\begin{array}{l}\text { Participant- } \\
\text { identified }^{*}\end{array}$ & Protein & $\begin{array}{l}\mathrm{El}, \mathrm{Ca}, \mathrm{Fe} \text {, } \\
\text { vitamins } \mathrm{A} \\
\text { and } \mathrm{C} \text {, thiamin, } \\
\text { riboflavin, } \\
\text { niacin }\end{array}$ & Stratified by sex & $\begin{array}{l}\text { Snacks contributed significantly } \\
\text { to the percentage of the RDA } \\
\text { for protein (13.4-24.1 \%), Ca } \\
(9 \cdot 9-20 \cdot 2 \%) \text {, Fe (11.3-34.8 } \\
\% \text { ), vitamin } \mathrm{C}(13 \cdot 5-29 \%), \\
\text { thiamin }(12-18 \%) \text {, riboflavin } \\
(12.4-24 \cdot 7 \%) \text { and niacin } \\
\text { (14.2-30\%). The contribution } \\
\text { of snacks to women's Fe } \\
\text { intakes was important as } \\
\text { meals only provided about } \\
57.5 \% \text { of the RDA }\end{array}$ \\
\hline $\operatorname{Kim}(2010)^{(83)}$ & $\begin{array}{r}\text { Korea } \\
\text { C/S }\end{array}$ & $\begin{array}{l}292 \text { men and } 391 \\
\text { women, 20-65 } \\
\text { years }\end{array}$ & $\begin{array}{l}\text { Meal and snack } \\
\text { frequency. Combi- } \\
\text { nations of meals } \\
\text { and snacks }\end{array}$ & $24 \mathrm{HR}$ & $\begin{array}{l}\text { Participant- } \\
\text { identified and } \\
\text { time-of-day }{ }^{\star}\end{array}$ & Protein, fat, $\mathrm{CHO}$ & $\mathrm{El}$ & Stratified by sex & $\begin{array}{l}\text { Absolute energy and } \mathrm{CHO} \text { intake } \\
\text { highest in the three meals } \\
\text { plus three snacks group. } \\
\text { There were no differences in } \\
\text { protein or fat intakes between } \\
\text { more frequent snackers } v \text {. } \\
\text { less frequent snackers }\end{array}$ \\
\hline Kuroda $(2013)^{(71)}$ & $\begin{array}{r}\text { Japan } \\
\mathrm{C} / \mathrm{S}\end{array}$ & $\begin{array}{l}275 \text { women students, } \\
19-25 \text { years }\end{array}$ & Meal skipping & $\begin{array}{l}\text { Diet: diet history } \\
\text { Q } \\
\text { Meal patterns: } \\
\text { Q }\end{array}$ & $\begin{array}{l}\text { Participant- } \\
\text { identified }\end{array}$ & Protein, fat, $\mathrm{CHO}$ & $\begin{array}{l}\mathrm{El}, \mathrm{Ca} \text {, } \\
\text { phosphate, } \\
\text { vitamins D } \\
\text { and K }\end{array}$ & - & $\begin{array}{l}\text { Skipping any meal was nega- } \\
\text { tively correlated with total EI } \\
(P<0.05) \text {. Skipping breakfast } \\
\text { was negatively correlated with } \\
\text { the absolute intake of all nutri- } \\
\text { ents examined }(P<0.05) \text {. } \\
\text { Skipping lunch or supper was } \\
\text { negatively correlated } \\
(P<0.05) \text { with absolute } \mathrm{CHO} \\
\text { intake and vitamin } \mathrm{K} \text { (lunch } \\
\text { only) and vitamin D intake } \\
\text { (supper only) }\end{array}$ \\
\hline $\operatorname{Min}(2011)^{(64)}$ & $\begin{array}{r}\text { Korea } \\
\text { C/S }\end{array}$ & $\begin{array}{l}118 \text { men and } 297 \\
\text { women, } 30-50 \\
\text { years }\end{array}$ & Breakfast skipping & $\begin{array}{l}1 \times 24 \mathrm{HR} \text { and } 2 \mathrm{~d} \\
\mathrm{FR} \text { (also } \\
\text { included a } \\
\text { weekend day) }\end{array}$ & Time-of-day* & $\mathrm{CHO}$, protein, fat & $\begin{array}{l}\text { Cholesterol, fibre, } \\
\text { Ca, P, Fe, Na, } \\
\text { K, Zn, folate, } \\
\text { vitamins A, C, } \\
\mathrm{E}_{\mathrm{E}, \mathrm{B}_{1}, \mathrm{~B}_{2} \text { and }} \\
\mathrm{B}_{3}\end{array}$ & Age, sex and $\mathrm{El}$ & $\begin{array}{l}\text { Those who skipped breakfast on } \\
\text { two or more of the days (rare } \\
\text { breakfast eaters) had lower } \\
\text { total El, fibre, } \mathrm{Ca}, \mathrm{CHO} \text { and } \mathrm{K} \text {, } \\
\text { but higher fat and Fe intakes. } \\
\text { Prevalence of not meeting the } \\
\text { EAR for } \mathrm{Ca} \text {, vitamin } \mathrm{C} \text { and } \\
\text { folate was significantly higher } \\
\text { among rare breakfast eaters, } \\
\text { compared with regular break- } \\
\text { fast eaters }\end{array}$ \\
\hline
\end{tabular}




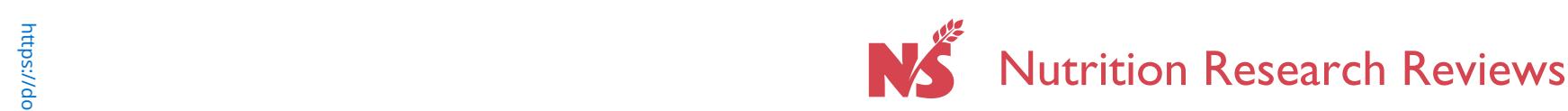

Table 3. Continued

\begin{tabular}{|c|c|c|c|c|c|c|c|c|c|}
\hline First author (year) & $\begin{array}{l}\text { Country } \\
\text { and study } \\
\text { design }\end{array}$ & Sample & $\begin{array}{l}\text { Aspect(s) of meal } \\
\text { patterns } \\
\text { examined }\end{array}$ & $\begin{array}{l}\text { Diet and meal } \\
\text { pattern } \\
\text { measure }\end{array}$ & $\begin{array}{l}\text { Meal or snack } \\
\text { definition }\end{array}$ & Macronutrients & $\begin{array}{l}\text { Other dietary } \\
\text { components }\end{array}$ & Covariates & Selected key findings \\
\hline Williams $(2005)^{(69)}$ & $\begin{array}{c}\text { Australia } \\
\text { C/S }\end{array}$ & $\begin{array}{l}5081 \text { men and } 5770 \\
\text { women, } \geq 19 \\
\text { years }\end{array}$ & Breakfast skipping & $\begin{array}{l}\text { 24HR } \\
\text { Meal patterns: } \\
\text { Q (one item) }\end{array}$ & $\begin{array}{l}\text { Participant- } \\
\text { identified }\end{array}$ & $\begin{array}{l}\text { Fat, } \mathrm{CHO} \text {, sugar, } \\
\text { protein }\end{array}$ & $\begin{array}{l}\text { El, fibre choles- } \\
\text { terol, vitamins } \\
\mathrm{A}, \mathrm{C} \text {, and } \mathrm{E}, \\
\text { thiamin, ribo- } \\
\text { flavin, niacin, } \\
\text { folate, } \mathrm{Zn}, \mathrm{Ca} \text {, } \\
\mathrm{Fe}, \mathrm{K} \text { and } \mathrm{P}\end{array}$ & Stratified by sex & $\begin{array}{l}\text { Compared with breakfast skip- } \\
\text { pers, those who ate breakfast } \\
\text { regularly }(\geq 5 \text { times } / \text { week) } \\
\text { had higher mean daily intakes } \\
\text { for all nutrients and minerals } \\
\text { examined except for fat } \\
(P<0 \cdot 05) \text {. In older adult ( } \geq 65 \\
\text { years) breakfast skippers, the } \\
\text { prevalence of not meeting } 70 \\
\% \text { of the RDI for almost all } \\
\text { nutrients was twice that of } \\
\text { regular breakfast eaters }\end{array}$ \\
\hline Winkler $(1999)^{(81)}$ & $\begin{array}{l}\text { Germany } \\
\mathrm{C} / \mathrm{S}\end{array}$ & $\begin{array}{l}899 \text { men, } 45-64 \\
\text { years }\end{array}$ & $\begin{array}{l}\text { Eating frequency dis- } \\
\text { tribution of nutrition } \\
\text { intake across EO }\end{array}$ & $7 \mathrm{dFR}$ & $\begin{array}{l}\text { Participant- } \\
\text { identified and } \\
\text { time-of-day }\end{array}$ & Protein, fat, $\mathrm{CHO}$ & $\begin{array}{l}\text { El, fibre, Ca, } \\
\text { alcohol }\end{array}$ & - & $\begin{array}{l}\text { On } 85.2 \% \text { of reported days, } \\
\text { dinner provided most of the } \\
\text { energy. Macronutrient intake } \\
\text { was contributed mostly by } \\
\text { meals and alcohol was mostly } \\
\text { drunk at dinner. Snacks in the } \\
\text { afternoon or late in the day } \\
\text { contained less protein and } \\
\text { fibre than the morning snack }\end{array}$ \\
\hline Zizza $(2001)^{(57)}$ & $\begin{array}{l}\text { USA } \\
\text { C/S (survey } \\
\text { years: } \\
\text { 1977-1978, } \\
\text { 1989-1991 } \\
\text { and } \\
\text { 1994-1995) }\end{array}$ & $\begin{array}{l}3789 \text { men and } 4706 \\
\text { women, } 19-29 \\
\text { years }\end{array}$ & $\begin{array}{l}\text { Snacking ( } v \text {. non- } \\
\text { snacking) }\end{array}$ & $\begin{array}{l}\text { 1997-1991: } \\
1 \times 24 \mathrm{HR} \text { and } \\
2 \mathrm{~d} \text { FR; } 1994- \\
\text { 1995: } \\
2 \times 24 \mathrm{HR}\end{array}$ & $\begin{array}{l}\text { Participant- } \\
\text { identified }{ }^{*}\end{array}$ & $\begin{array}{l}\text { Protein, fat, } \mathrm{CHO} \text {, } \\
\text { SFA }\end{array}$ & $\mathrm{El}$ & - & $\begin{array}{l}\text { Within each survey, compared } \\
\text { with non-snackers, snackers } \\
\text { had significantly higher intakes } \\
\text { of CHO, fat and SFA } \\
(P<0.01) \text {. In the } 1994-1996 \\
\text { survey, snackers had signifi- } \\
\text { cantly greater protein intakes } \\
\text { than non-snackers }(P<0 \cdot 01) \text {. } \\
\text { However, there were no sig- } \\
\text { nificant differences when all } \\
\text { the above macronutrients } \\
\text { were expressed as percen- } \\
\text { tage of El }\end{array}$ \\
\hline Zizza $(2007)^{(77)}$ & $\begin{array}{l}\text { USA } \\
\text { C/S }\end{array}$ & $\begin{array}{l}2002 \text { men and } \\
\text { women, } \geq 65 \\
\text { years }\end{array}$ & $\begin{array}{l}\text { Snacking ( } v \text {. non } \\
\text { snacking) }\end{array}$ & $24 \mathrm{HR}$ & $\begin{array}{l}\text { Participant- } \\
\text { identified }\end{array}$ & $\begin{array}{l}\text { Protein, CHO, fat, } \\
\text { SFA }\end{array}$ & El, alcohol & $\begin{array}{l}\text { Age, poverty } \\
\text { income ratio, } \\
\text { sex, race, edu- } \\
\text { cation, marital } \\
\text { status, smoking }\end{array}$ & $\begin{array}{l}\text { Snackers had significantly higher } \\
\text { intakes of energy, protein, } \\
\text { CHO, fat and SFA, compared } \\
\text { with non-snackers }(P<0.05) \text {. } \\
\text { Snacking contributed approxi- } \\
\text { mately } 25 \% \text { of daily energy } \\
\text { and CHO intakes, and } 20 \text { and } \\
12 \% \text { of daily fat and protein } \\
\text { intakes, respectively }\end{array}$ \\
\hline Zizza $(2010)^{(70)}$ & $\begin{array}{l}\text { USA } \\
\text { C/S }\end{array}$ & $\begin{array}{l}2056 \text { men and } \\
\text { women, } \geq 65 \\
\text { years }\end{array}$ & Snack frequency & $2 \times 24 \mathrm{HR}$ & $\begin{array}{l}\text { Participant- } \\
\text { identified }\end{array}$ & & $\begin{array}{l}\text { Vitamins } \mathrm{A}, \mathrm{B}_{6}, \\
\mathrm{~B}_{12}, \mathrm{C}, \mathrm{E} \text { and } \\
\mathrm{K} \text {, folate, nia- } \\
\text { cin, } \beta \text {-caro- } \\
\text { tene, } \mathrm{Cu}, \\
\text { lycopene, } \mathrm{Fe}, \\
\mathrm{Ca}, \mathrm{Zn}, \mathrm{P}, \mathrm{K} \\
\mathrm{Se}\end{array}$ & $\begin{array}{l}\text { El, sex, race, } \\
\text { education, } \\
\text { income, BMI }\end{array}$ & $\begin{array}{l}\text { With increasing snack frequency, } \\
\text { mean daily intakes of vitamins } \\
\mathrm{A}, \mathrm{C} \text { and } \mathrm{E}, \beta \text {-carotene, } \mathrm{Mg}, \\
\mathrm{Cu} \text { and } \mathrm{K} \text { significantly } \\
\text { increased, whereas Se } \\
\text { intakes significantly decreased } \\
(P<0.05)\end{array}$ \\
\hline
\end{tabular}

EO, eating occasion; FR, food record; CHO, carbohydrate; El, energy intake; C/S, cross-sectional; 24HR, $24 \mathrm{~h}$ recall; PA, physical activity; $\mathrm{Q}$, questionnaire; RDA, recommended daily allowance; EAR, estimated average requirement; $\mathrm{RDI}$, recommended daily intake.

Beverages could qualify as a separate eating occasion.

Energy misreporters or under-reporters excluded from analyses.

Milk in excess of 0.5 pints $(284 \mathrm{ml})$ was the only beverage that could qualify as a separate eating occasion. 
difference in definitions adopted across these studies. Interestingly, two studies ${ }^{(36,81)}$ reported that snacks provided a greater percentage of total sugars but not total carbohydrate than meals, and Winkler et al ${ }^{(81)}$ noted that snacks eaten after lunchtime contained less protein and fibre than the morning snack. Two studies ${ }^{(73,79)}$ showed that the percentage of protein intake was highest in the evening, particularly among older adults ${ }^{(73)}$. This suggests that macronutrient differences between meals and snacks may be influenced by both the type and timing of food consumed.

There is a paucity of information on the relative contributions of meals or snacks to intakes of micronutrients and other dietary components. Roos \& Prättälä ${ }^{(65)}$ examined the impact of adherence to a conventional Finnish meal pattern (breakfast, warm lunch and warm dinner plus two snacks) among 1861 adults aged 25-64 years and found, per unit of energy, that meals contributed more fibre and carotenoids but less sugar, vitamin $\mathrm{C}$ and alcohol than snacks. This finding remained consistent across sex and after adjustment for age and region Additionally, a study on the adherence to a Dutch meal pattern (breakfast, morning snack, lunch bread meal, afternoon snack, hot dinner meal) ${ }^{(30)}$ found that the (hot) dinner meal was the main contributor to the intakes of (haem) Fe, Zn and vitamins $B_{1}, B_{6}, B_{12}, C, D$ and E. Snacks may also be important in assisting populations to meet dietary guidelines for micronutrient intakes; one study ${ }^{(75)}$ of young adult students found that snacks contributed significantly to the percentage of the recommended daily allowances for $\mathrm{Ca}, \mathrm{Fe}$, vitamin $\mathrm{C}$, thiamin, riboflavin and niacin. In the same study, snacks were important contributors of $\mathrm{Fe}$ and $\mathrm{Ca}$ intake among women, whose meal contributions of these micronutrients were only about $65 \%$ and about $79 \%$ of the RDA, respectively.

\section{Eating frequency and nutrient intakes}

Of eighteen studies that examined eating frequency (including snacking frequency), fourteen found that eating frequency was associated with higher $\mathrm{EI}^{(40,41,45-47,52,57,67,72,77,78,80,82,83)}$. However, the evidence to support associations with nutrient intake is less consistent. Two large population-based studies, one in US adults $^{(52)}$ and the other in Swedish adults ${ }^{(80)}$, found that those who ate six or more times per $\mathrm{d}$ had higher intakes of carbohydrate and fibre but lower intakes of fat and protein compared with adults who ate once or twice per $\mathrm{d}$ or less than three times per $\mathrm{d}$, respectively. In these studies, a higher eating frequency was also associated with higher nutrient densities of folate, vitamin $\mathrm{C}$ and $\mathrm{Fe}^{(52,80)}$, and $\mathrm{Ca}$ and $\mathrm{K}^{(52)}$. Adjustment for multiple important sociodemographic and lifestyle-related confounders ${ }^{(52)}$ and exclusion of energy misreporters ${ }^{(80)}$ did not attenuate the significance of the results in these two studies.
Snacking frequency also appears to be an important contributor to intakes of macro- and micronutrients among older US adults aged $\geq 65$ years ${ }^{(70,77)}$. Snackers (consumers of one or more snacks per d) had significantly higher intakes of protein, carbohydrate and fat compared with non-snackers ${ }^{(77)}$, and a higher snacking frequency was associated with higher mean daily intakes of vitamins $\mathrm{A}, \mathrm{C}$ and $\mathrm{E}, \beta$-carotene, $\mathrm{Mg}$ and $\mathrm{K}^{(70)}$, after controlling for important confounders in both of these studies.

In contrast, three studies ${ }^{(45,46,67)}$ found that the proportion of energy from protein but not fat was negatively associated with snacking frequency. Additionally, Ovaskainen $e t a l{ }^{(47)}$ found that men with a snack-dominated meal pattern (defined as the majority of daily EI derived from snacks) had significantly lower fibre and micronutrient intake (vitamins $\mathrm{A}, \mathrm{C}, \mathrm{E}, \mathrm{Ca}, \mathrm{K}, \mathrm{Na}, \mathrm{Fe}, \mathrm{Mg}$ ) when compared with men with a meal-dominated pattern. The inconsistency in findings may be partly explained by how snacks have been examined relative to the overall eating pattern: that is, snack consumption in addition to main meals $v$. snacking in place of meals.

\section{Meal skipping and nutrient intakes}

A total of six studies ${ }^{(23,64,66,68,69,71)}$ were identified that examined the influence of breakfast skipping on nutrient intakes and only one study ${ }^{(71)}$ was identified that examined the nutritional impact of omitting the lunch or dinner meal. Breakfast skipping was consistently associated with lower micronutrient intakes ${ }^{(23,64,66,68,69,71)}$, even after adjustment for EI and other important confounders ${ }^{(23,60,66)}$. Breakfast skipping was also associated with a higher prevalence of not meeting the recommended intakes for $\mathrm{Ca}^{(64,66,68,69)}$, vitamin $C^{(23,64,68,69)}$, folate ${ }^{(64,68,69)}$, vitamin $A^{(23,66,68,69)}$ and $\mathrm{Mg}^{(23,66)}$ compared with regular breakfast consumers. In addition, Williams ${ }^{(69)}$ found that, among older Australian adults (aged $\geq 65$ years), the prevalence of not meeting the recommended daily intakes for almost all nutrients examined was, among breakfast skippers, twice that of regular breakfast eaters. In a study of Japanese women students ${ }^{(71)}$, skipping lunch or supper was negatively correlated $(P<0.05)$ with total EI and absolute intakes of carbohydrate and vitamin K (lunch only).

\section{Meal timing and nutrient intakes}

Only three studies were identified that examined associations between meal timing and $\mathrm{EI}^{(76,79,84)}$ or macronutrient intake ${ }^{(76)}$. In these studies, the proportion of $\mathrm{EI}$ consumed in the evening was positively associated with overall $\mathrm{EI}^{(76,79,84)}$. Among a large sample of US men and women, an increasing proportion of energy consumed after 17.00 hours was associated with an increase in mean daily alcohol intake but a decrease in mean carbohydrate intake $(P<0 \cdot 05)$. 


\section{Studies examining meal patterns and overall diet quality}

A total of fourteen studies were identified that examined associations between meal patterns and measures of overall diet quality (Table 4). Most studies were conducted in the USA ${ }^{(23,28,85-89)}$, with fewer studies conducted in Australia $^{(22,56,90)}$, Canada ${ }^{(91,92)}$, Western Europe ${ }^{(93)}$ and Iran $^{(94)}$. Of the studies, seven ${ }^{(22,28,56,85-88)}$ used bivariate analyses to determine whether diet quality was associated with meal patterns with the purpose of identifying its role as covariate in the relationship between meal patterns and health outcomes. Meal patterns were mostly assessed using a participant-identified approach ${ }^{(23,56,85,86,88-91)}$; however, the methods used to measure participants' EO also varied across these studies. For example, some studies asked participants to report their EO in response to one or two questionnaire items ${ }^{(85,86,88,90,91)}$ whereas other studies used $24 \mathrm{~h}$ recall methodology $(23,89)$. Importantly, where questionnaire items were used, the reliability and/or validity of these measures were rarely reported ${ }^{(56)}$. Additionally, in three studies ${ }^{(87,92,93)}$ the approach used to define a 'meal' could not be readily identified.

The most common measure used to assess overall diet quality was a previously validated and reliability tested a priori diet quality index which reflects an individual's adherence to the dietary guidelines for the country of the sample population (for example, the Healthy Eating Index (HEI), the Alternative HEI (AHEI) and the Dietary Guidelines Index (DGI) $)^{(23,28,86,88-90,92,94)}$. The measures used in the remaining studies were varied and included scores that measured adherence to: a traditional Mediterranean diet (MEDAS score) ${ }^{(93)}$; Dietary Approaches to Stop Hypertension (DASH) diet score ${ }^{(28)}$; a dietary approaches to prevent heart disease diet score (Optimal Macronutrient Intake Trial to Prevent Heart Disease (OmniHeart) score $)^{(5)}$; hypothesised healthy eating patterns (a priori diet score $)^{(87)}$; and national guidelines for healthy eating $^{(22,56,91)}$. The associations between these diet quality measures and different meal patterns are discussed below.

\section{Eating frequency and diet quality}

Few studies have examined associations between eating frequency (including meal and/or snack frequency) and diet quality. Among US male health professionals, a higher eating frequency was associated with higher DASH scores, reflecting higher diet quality $(r$ 0.14; no $P$ value provided $)^{(85)}$. A higher meal frequency was also associated with higher diet quality as measured by the Canadian HEI among older male and female adults aged 67-84 years old (men: $\beta$ 1.91, $P<0.02$; women: $\beta 3.61$, $P<0.0001)^{(91)}$. Of note, neither of these studies adjusted for total EI. The mean score for HEI-2005 also increased with increased daily snacking frequency (for example, no snacks $=49.3(\operatorname{se} 0.5) \quad v \geq 4$ snacks $=51.5(\operatorname{se} 0.6)$, $P<0.001)$ among US adults, after adjustment for sociodemographic factors, BMI, eating three or more meals daily and EI from meals ${ }^{(89)}$. Conversely, another study reported no association between snacking between meals and diet quality ${ }^{(91)}$, and Kim \& $\mathrm{Kim}^{(83)}$ found that a HEI score significantly decreased according to each increased quartile of a snack-dominant eating score (high snack frequency and low meal frequency) $(P<0 \cdot 01)$. Again, neither study reported adjustment for total EI.

\section{Meal skipping/regularity and diet quality}

Of the nine studies identified that examined associations between skipping breakfast and diet quality, six found a negative association ${ }^{(22,23,86,87,90,94)}$ and three found no association $^{(28,91,93)}$. However, the lack of association in two of these latter studies may be explained by their respective study populations; overall diet quality was high among men in the US Health Professionals Followup study ${ }^{(28)}$ while Dewolfe \& Millan $^{(91)}$ used a small convenience sample of eighty-four female and twenty-one male older adults from a single region in Canada. In the latter study ${ }^{(91)}$, eating lunch daily was associated with higher diet quality scores that assessed compliance with the Canadian Guide to Healthy Eating. No other studies were identified that have examined skipping/regularity of meals other than breakfast.

\section{Meal timing and diet quality}

Studies examining associations between meal timing and diet quality are rare. In the US Health Professionals Follow-up study, Cahill et $a l^{(28)}$ found no association between late night eating (defined as eating after going to bed) and AHEI scores; however, as mentioned previously, the authors acknowledged that AHEI scores were high in this sample, irrespective of their reported meal patterns.

\section{Potential impact of different meal definitions on the characterisation of meal patterns and associations with nutrient intakes and diet quality}

Clear and objective definitions of what is a meal and what is a snack are critical for determining the energy and nutrient contributions of meals $v$. snacks, meal skipping or meal timing. Without a clear definition misclassification bias is likely, thus affecting the interpretation of associations with nutrients both within and across studies. In allowing participants to identify meals and snacks, subjective decision-making is inherently present. Previous research suggests that situational cues such as the type, quality or amount of food and the presence of others may affect a participant's decision to classify an EO as a meal or a snack $^{(95)}$. It is also unclear whether the same meal or snack situation would be classified similarly by different individuals; research in this area is needed in order to 
Table 4. Characteristics of studies that have examined associations between meal patterns and overall diet quality

\begin{tabular}{|c|c|c|c|c|c|c|c|c|}
\hline First author (year) & $\begin{array}{l}\text { Country } \\
\text { and study } \\
\text { design }\end{array}$ & Sample & $\begin{array}{l}\text { Aspect(s) of } \\
\text { meal } \\
\text { patterns } \\
\text { examined } \\
\end{array}$ & $\begin{array}{l}\text { Measure(s) } \\
\text { to assess diet and } \\
\text { meal patterns }\end{array}$ & $\begin{array}{l}\text { Meal or snack } \\
\text { definition }\end{array}$ & $\begin{array}{l}\text { Diet quality } \\
\text { indicator(s) }\end{array}$ & Covariates & Selected key findings \\
\hline Azadbakht (2013) $)^{(94)}$ & $\begin{array}{l}\text { Iran } \\
\text { C/S }\end{array}$ & $\begin{array}{l}411 \text { women } \\
\text { students, } \\
18-28 \text { years }\end{array}$ & $\begin{array}{l}\text { Breakfast } \\
\text { skipping }\end{array}$ & $\begin{array}{l}\text { FFQ } \\
\text { Meal patterns: not } \\
\text { described }\end{array}$ & Time of-day* & HEI, DDS & $\begin{array}{l}\text { Not clear if } \\
\text { covariates } \\
\text { were adjusted } \\
\text { for in the } \\
\text { multivariate } \\
\text { ANOVA }\end{array}$ & $\begin{array}{l}\text { HEI and DDS scores and } \\
\text { diversity scores for fruits, } \\
\text { vegetables and whole } \\
\text { grains were significantly } \\
\text { lower among breakfast } \\
\text { skippers than consumers } \\
(P<0.001)\end{array}$ \\
\hline $\begin{array}{l}\text { Deshmukh-Taskar } \\
(2010)^{(23)}\end{array}$ & $\begin{array}{l}\text { USA } \\
\text { C/S }\end{array}$ & $\begin{array}{l}2615 \text { men and } \\
\text { women, } \\
20-39 \text { years }\end{array}$ & $\begin{array}{l}\text { Breakfast } \\
\text { skipping }\end{array}$ & $1 \times 24 \mathrm{HR}$ & $\begin{array}{l}\text { Participant- } \\
\text { identified }^{*}\end{array}$ & $\mathrm{HEI}$ & $\begin{array}{l}\text { Ethnicity, sex, } \\
\text { sex } \times \\
\text { ethnicity, age, } \\
\text { poverty } \\
\text { income ratio, } \\
\text { smoking } \\
\text { status, marital } \\
\text { status and } \\
\text { PA }\end{array}$ & $\begin{array}{l}\text { Breakfast skippers had } \\
\text { significantly lower } \\
(P<0.0001) \text { HEl scores } \\
\text { than those who consumed } \\
\text { ready-to-eat breakfast cer- } \\
\text { eals or other breakfast } \\
\text { foods }\end{array}$ \\
\hline Dewolfe $(2003)^{(91)}$ & $\begin{array}{c}\text { Canada } \\
\text { C/S }\end{array}$ & $\begin{array}{l}84 \text { men and } 21 \\
\text { women, } \geq 65 \\
\text { years }\end{array}$ & $\begin{array}{l}\text { Meal skipping } \\
\text { and snacking }\end{array}$ & $\begin{array}{l}3 \times 24 \mathrm{HR} \\
\quad \text { Meal patterns: } \mathrm{Q}\end{array}$ & $\begin{array}{c}\text { Participant- } \\
\text { identified }\end{array}$ & $\begin{array}{l}\text { Diet score based on } \\
\text { compliance with } \\
\text { national dietary } \\
\text { guidelines }\end{array}$ & $\begin{array}{l}\text { Preparing own } \\
\text { meals, how } \\
\text { well food } \\
\text { tastes, } \\
\text { prescription } \\
\text { medication } \\
\text { use, sex }\end{array}$ & $\begin{array}{l}\text { Eating lunch daily was posi- } \\
\text { tively associated (standar- } \\
\text { dised } \beta=0.24,95 \% \mathrm{Cl} \\
0.05,0.42 \text { ) with the diet } \\
\text { score reflecting adherence } \\
\text { to Canadian dietary guide- } \\
\text { lines }\end{array}$ \\
\hline Cahill (2013) & $\begin{array}{l}\text { USA } \\
\text { Prospective } \\
\text { (16-year follow-up) }\end{array}$ & $\begin{array}{l}29209 \text { health } \\
\text { professional } \\
\text { men, } 40-75 \\
\text { years }\end{array}$ & $\begin{array}{l}\text { Breakfast eating } \\
\text { and late-night } \\
\text { eating }\end{array}$ & $\begin{array}{l}\text { FFQ† } \\
\quad \text { Meal patterns: } \mathrm{Q}\end{array}$ & Time-of-day & AHEI-2010 & - & $\begin{array}{l}\text { Based on age-standardised } \\
\text { baseline data, no signifi- } \\
\text { cant differences in AHEI } \\
\text { scores were reported } \\
\text { between breakfast consu- } \\
\text { mers and non-breakfast } \\
\text { consumers or late-night } \\
\text { eaters and non-late-night } \\
\text { eaters }\end{array}$ \\
\hline $\operatorname{Kim}(2011)^{(88)}$ & $\begin{array}{l}\text { USA/Puerto Rico } \\
\text { C/S }\end{array}$ & $\begin{array}{r}27983 \text { women, } \\
35-74 \text { years }\end{array}$ & $\begin{array}{l}\text { Snack domi- } \\
\text { nance and } \\
\text { conventional } \\
\text { eating pattern }\end{array}$ & $\begin{array}{l}\text { Modified block FFQ† } \\
\text { Meal patterns: } Q\end{array}$ & $\begin{array}{c}\text { Participant- } \\
\text { identified }\end{array}$ & HEI & - & $\begin{array}{l}\text { A higher conventional eating } \\
\text { score (eating meals and } \\
\text { snacks during convention- } \\
\text { al times) was associated } \\
\text { with higher HEI scores } \\
(P<0.01) \text { whereas a } \\
\text { higher snack-dominant } \\
\text { eating score was associ- } \\
\text { ated with lower HEl } \\
\text { scores }(P<0.01)\end{array}$ \\
\hline Mekary (2012) ${ }^{(85)}$ & $\begin{array}{l}\text { USA } \\
\text { Prospective } \\
\text { (14-year follow-up) }\end{array}$ & $\begin{array}{l}34968 \text { men, } \\
40-75 \text { years }\end{array}$ & $\begin{array}{l}\text { Eating } \\
\text { frequency }\end{array}$ & $\begin{array}{l}\text { FFQ† } \\
\quad \text { Meal patterns: } \mathrm{Q}\end{array}$ & $\begin{array}{l}\text { Participant- } \\
\text { identified }\end{array}$ & DASH score & - & $\begin{array}{l}\text { Based on age-standardised } \\
\text { baseline data, there was a } \\
\text { positive association } \\
\text { between eating frequency } \\
\text { and the DASH score } \\
(r 0.14)\end{array}$ \\
\hline Mekary $(2013)^{(86)}$ & $\begin{array}{l}\text { USA } \\
\text { Prospective } \\
\text { (6-year follow-up) }\end{array}$ & 46289 women & $\begin{array}{l}\text { Eating breakfast } \\
\text { regularly } \\
\text { and eating } \\
\text { frequency }\end{array}$ & $\begin{array}{l}\text { FFQ† } \\
\text { Meal patterns: Q } \\
\text { (two items) }\end{array}$ & $\begin{array}{l}\text { Participant- } \\
\text { identified }^{*}\end{array}$ & AHEI-2010 & - & $\begin{array}{l}\text { Based on age-standardised } \\
\text { baseline data, women } \\
\text { who ate breakfast } \leq 6 \\
\text { times/week had lower } \\
\text { scores for the AHEI-2010 } \\
\text { than regular breakfast } \\
\text { consumers. Diet quality by } \\
\text { eating frequency was not } \\
\text { assessed }\end{array}$ \\
\hline
\end{tabular}


Table 4. Continued

\begin{tabular}{|c|c|c|c|c|c|c|c|c|}
\hline First author (year) & $\begin{array}{l}\text { Country } \\
\text { and study } \\
\text { design }\end{array}$ & Sample & $\begin{array}{l}\text { Aspect(s) of } \\
\text { meal } \\
\text { patterns } \\
\text { examined }\end{array}$ & $\begin{array}{l}\text { Measure(s) } \\
\text { to assess diet and } \\
\text { meal patterns }\end{array}$ & $\begin{array}{l}\text { Meal or snack } \\
\text { definition }\end{array}$ & $\begin{array}{l}\text { Diet quality } \\
\text { indicator(s) }\end{array}$ & Covariates & Selected key findings \\
\hline Mesas $(2012)^{(93)}$ & $\begin{array}{r}\text { Spain } \\
\text { C/S }\end{array}$ & $\begin{array}{l}10791 \text { men and } \\
\text { women, } \geq 18 \\
\text { years }\end{array}$ & $\begin{array}{l}\text { Skipping } \\
\text { breakfast }\end{array}$ & $\begin{array}{l}\text { Diet history } \mathrm{Q} \dagger \\
\text { Meal patterns: } \mathrm{Q}\end{array}$ & $\begin{array}{l}\text { Never eating } \\
\text { anything at } \\
\text { the breakfast } \\
\text { occasion } \\
\text { (meal defi- } \\
\text { nition could } \\
\text { not be estab- } \\
\text { lished) }\end{array}$ & $\begin{array}{l}\text { MEDAS score; the } \\
\text { OmniHeart diet } \\
\text { score }\end{array}$ & $\begin{array}{l}\text { Age, sex, edu- } \\
\text { cation, social } \\
\text { class, smok- } \\
\text { ing, alcohol, } \\
\text { binge drink- } \\
\text { ing, PA at } \\
\text { work, BMl } \\
\text { and morbidity }\end{array}$ & $\begin{array}{l}\text { No significant associations } \\
\text { were found between skip- } \\
\text { ping breakfast and either } \\
\text { the MEDAS score or the } \\
\text { OmniHeart diet score }\end{array}$ \\
\hline Odegaard $(2013)^{(87)}$ & $\begin{array}{l}\text { USA } \\
\text { Prospective } \\
\text { (follow-up: } 18 \text { years) }\end{array}$ & $\begin{array}{l}3598 \text { men and } \\
\text { women, } \\
18-30 \text { years } \\
\text { at baseline }\end{array}$ & $\begin{array}{l}\text { Breakfast } \\
\text { frequency }\end{array}$ & $\begin{array}{l}\text { Diet history } \mathrm{Q} \dagger \\
\quad \text { Meal patterns: } \mathrm{Q}\end{array}$ & $\begin{array}{l}\text { No definition } \\
\text { provided }\end{array}$ & $\begin{array}{l}\text { A priori diet quality } \\
\text { score (no specific } \\
\text { name given) }\end{array}$ & - & $\begin{array}{l}\text { Based on C/S data at the } \\
\text { 7-year follow-up, higher } \\
\text { levels of breakfast intakes } \\
\text { were associated with } \\
\text { higher diet quality scores }\end{array}$ \\
\hline Shatenstein $(2013)^{(92)}$ & $\begin{array}{c}\text { Canada } \\
\text { C/S }\end{array}$ & $\begin{array}{l}853 \text { men and } \\
940 \text { women, } \\
67-84 \text { years }\end{array}$ & $\begin{array}{l}\text { Meal frequency } \\
\text { (snacks not } \\
\text { included) }\end{array}$ & $\begin{array}{l}3 \times 24 \mathrm{HR} \\
\text { Meal patterns: } \mathrm{Q}\end{array}$ & $\begin{array}{c}\text { No definition } \\
\text { provided }\end{array}$ & Canadian HEI & $\begin{array}{l}\text { Sex-specific } \\
\text { models. } \\
\text { Inclusion of } \\
\text { the following } \\
\text { covariates } \\
\text { depended on } \\
\text { model: edu- } \\
\text { cation, diet, } \\
\text { income, alco- } \\
\text { hol, wears } \\
\text { dentures, per- } \\
\text { ceived physi- } \\
\text { cal health, } \\
\text { eats in res- } \\
\text { taurants, } \\
\text { nutrition } \\
\text { knowledge, } \\
\text { hunger, BMI, } \\
\text { chewing } \\
\text { problems }\end{array}$ & $\begin{array}{l}\text { Among males and females, } \\
\text { number of meals/d was } \\
\text { positively associated with } \\
\text { Canadian HEI scores } \\
(\beta=1.91, P<0.02 \text { and } \\
\beta=3.61, P<0.0001 \\
\text { respectively) }\end{array}$ \\
\hline Smith $(2010)^{(22)}$ & $\begin{array}{l}\text { Australia } \\
\text { Prospective }\end{array}$ & $\begin{array}{l}1020 \text { men and } \\
1164 \text { women, } \\
9-15 \text { years } \\
\text { at baseline } \\
\text { and } 26-36 \\
\text { years at } \\
\text { follow-up }\end{array}$ & $\begin{array}{l}\text { Breakfast skip- } \\
\text { ping }\end{array}$ & $\begin{array}{l}\text { FFQ } \\
\text { Meal patterns: Q } \\
\text { (meal patterns } \\
\text { chart) }\end{array}$ & $\begin{array}{l}\text { Participant- } \\
\text { identified and } \\
\text { time-of-day }\end{array}$ & $\begin{array}{l}\text { Compliance with } \\
\text { dietary advice in } \\
\text { the Australian } \\
\text { Guide to Healthy } \\
\text { Eating }\end{array}$ & - & $\begin{array}{l}\text { Participants who skipped } \\
\text { breakfast in both child- } \\
\text { hood and adulthood were } \\
\text { less likely to meet rec- } \\
\text { ommendations for fruit, } \\
\text { dairy products, lean meat } \\
\text { and alternatives and take- } \\
\text { out foods }(P<0.001) \text { than } \\
\text { those who did not skip } \\
\text { breakfast at either time } \\
\text { point }\end{array}$ \\
\hline Smith $(2012)^{(56)}$ & $\begin{array}{c}\text { Australia } \\
\mathrm{C} / \mathrm{S}\end{array}$ & $\begin{array}{l}1273 \text { men and } \\
1502 \text { women, } \\
26-36 \text { years }\end{array}$ & $\begin{array}{l}\text { Eating } \\
\text { frequency }\end{array}$ & $\begin{array}{l}\text { FFQ } \\
\text { Meal patterns: Q } \\
\text { (meal patterns } \\
\text { chart) }\end{array}$ & $\begin{array}{l}\text { Participant- } \\
\text { identified }\end{array}$ & $\begin{array}{l}\text { Diet score based on } \\
\text { compliance with } \\
\text { national dietary } \\
\text { guidelines }\end{array}$ & Stratified by sex & $\begin{array}{l}\text { There was a positive associ- } \\
\text { ation }(P<0.001) \text { between } \\
\text { daily eating frequency and } \\
\text { dietary scores, and meet- } \\
\text { ing recommendations for } \\
\text { fruit and dairy products } \\
\text { among both men and } \\
\text { women }\end{array}$ \\
\hline Smith $(2013)^{(90)}$ & $\begin{array}{c}\text { Australia } \\
\mathrm{C} / \mathrm{S}\end{array}$ & $\begin{array}{l}4123 \text { women } \\
\text { from low-SES } \\
\text { areas, } 18-45 \\
\text { years }\end{array}$ & $\begin{array}{l}\text { Breakfast } \\
\text { skipping }\end{array}$ & $\begin{array}{l}\text { FFQ } \\
\text { Meal patterns: Q } \\
\text { (one item) }\end{array}$ & $\begin{array}{l}\text { Participant- } \\
\text { identified }\end{array}$ & DGI & - & $\begin{array}{l}\text { Compared with women who } \\
\text { ate breakfast }<1 \mathrm{~d} / \text { week } \\
\text { or } 1-2 \mathrm{~d} / \text { week, those who } \\
\text { ate breakfast } \geq 3 \mathrm{~d} / \text { week } \\
\text { were more likely to be in } \\
\text { the highest tertile for DGl } \\
\text { scores }\end{array}$ \\
\hline
\end{tabular}




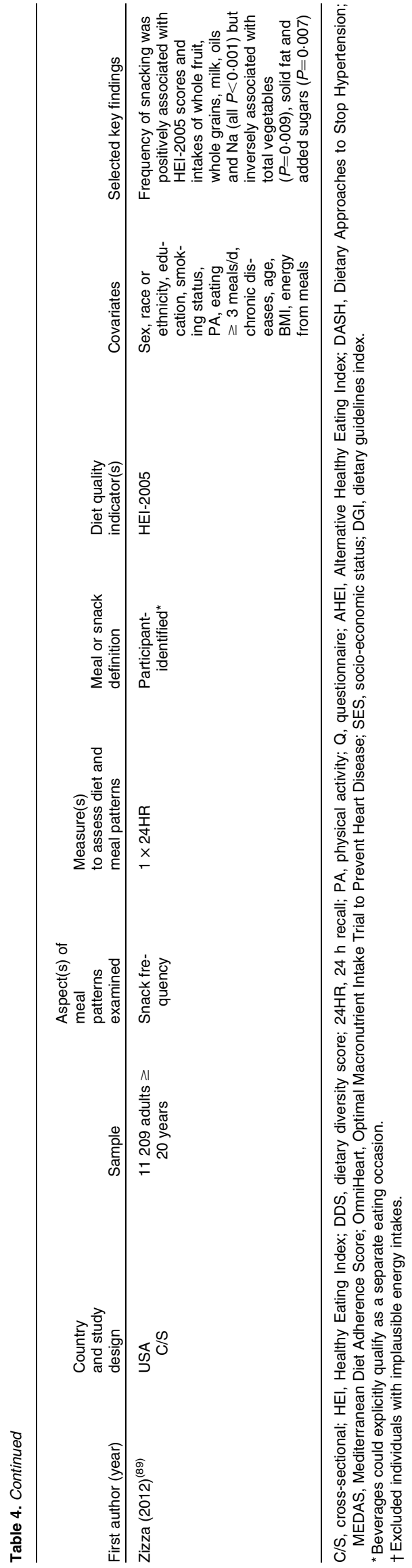

better understand the between-subject variation when applying a participant-identified definition. While a timeof-day approach can be applied consistently for all participants, it may not capture meals and snacks eaten at varied times. Furthermore, it is unknown whether meals and snacks would be classified similarly if either a participantidentified or a time-of-day approach were applied. Research on the comparability of the different definitions that seek to define meals and snacks would help address this issue.

It is important to note that studies that have examined eating frequency (including meal and/or snack frequency) differ in both the methods used to define meals and snacks and the time-gap to separate individual EO, which may make an impact on the frequency of the respective EO reported. For example, while most meal or snack definitions include beverages alongside food, not all studies explicitly considered a beverage-only occasion as a separate $\mathrm{EO}^{(40,41,67,72,78,82)}$. In addition, larger time intervals used to separate EO may result in EO, including beverage-only occasions, being overlooked and this may affect associations between eating frequency and energy and nutrient intakes. For example, Kant et al. ${ }^{(96)}$ demonstrated a positive association between $24 \mathrm{~h}$ beverage EI with saturated fat, sugar, $\mathrm{Na}$ and alcohol intakes, after adjustment for EI from foods. However, a definition that excludes 'lowenergy' beverage-only EO (for example, $<210 \mathrm{~kJ}$ ) may also be important. A recent study ${ }^{(5)}$ showed that, compared with a definition that included all energy-containing EO, there was a stronger correlation between eating frequency and EI after applying a definition that used a minimum energy criterion of $\geq 210 \mathrm{~kJ}$ (men: $r 0.45 v \cdot 0.53$; women $r 0.39 v \cdot 0.57$, respectively), which remained after excluding energy misreporters.

As few studies have examined associations between meal patterns and diet quality, the impact of different meal definitions is difficult to assess. Breakfast skipping was consistently inversely associated with diet quality in six out of nine studies, despite the different definitions used (time-of-day ${ }^{(88,94)}$ and participant identified $\left.^{(22,23,28,86,90)}\right)$, and in some cases, no clear definition was provided ${ }^{(87,93)}$.

Of note, many studies that have examined meal patterns and diet quality also used questionnaire items with unreported reliably and validity to collect meal pattern data. The lexical and semantic features of questionnaire items can differ between studies and may influence participant responses ${ }^{(97)}$. For example, items may ask participants to indicate the times of the day they usually eat ${ }^{(28)}$ or how many days they usually have something to eat for breakfast ${ }^{(90)}$, whereas other items provide additional instruction such as include all beverages ${ }^{(88)}$ or all nutritive beverages ${ }^{(86)}$. Questions that use the word 'eat' but provide no additional examples or cues as to what to include may therefore only elicit information about food-only EO or combined food and beverage EO but not beverage-only EO. However, 
until questionnaire items are validated against a preexisting valid method (for example, a $24 \mathrm{~h}$ recall), how accurately they capture meal, snack and all EO (including beverages) remains unclear.

\section{Discussion}

Meal patterns are multidimensional and can be described according to their patterning, format and context. However, due to the limited dietary assessment methods available, most research has focused on meal patterning. To date, a variety of definitions has been used to examine meal patterns. In addition, a number of additional criteria have been adopted in meal pattern research, which may have an impact on the types of meal patterns reported and described in the literature. Although over the past few decades there has been general consensus that a universally accepted definition of a meal is crucial ${ }^{(54,98)}$, there have been few attempts to define meals in a consistent and standardised way.

Research suggests that different meal and/or snack patterns are related to both nutrient intakes and overall diet quality, with the most consistent finding being an inverse relationship between skipping breakfast and nutrient intakes/diet quality. Skipping meals other than breakfast has rarely been examined but may be important, particularly for vulnerable groups such as the elderly ${ }^{(69,91)}$. In addition the nutritional impact of snack, meal and overall eating frequency remains unclear and little research has looked at the how meal timing influences nutritional intake/overall diet quality. This may be an important area of research in light of preliminary evidence suggesting that the timing of energy and/or macronutrient intake during the day is associated with cardiometabolic risk $^{(27,28,99,100)}$

The conflicting findings for the associations between eating frequency (including snack/meal frequency) and nutrient intake/diet quality may be, in part, attributed to not only the heterogeneity of meal patterns examined, but also to different definitions of meals and snacks. While meals and snacks are hypothesised to exert different effects on EI and nutrient intake, some researchers suggest that the sociocultural and value-laden nature of the terms used to identify different meals and snacks precludes such delineation ${ }^{(101)}$. Although it is widely acknowledged that different definitions used to define meals/snacks are likely to hamper interpretation of findings across studies $^{(8,44)}$, research explicitly examining the impact of these different definitions is rare ${ }^{(55)}$. There has been little attempt to examine meal patterns in a consistent and standardised way.

Another important consideration for future research examining eating frequency is potential overlap in the meal patterns that are being examined, which further complicates comparisons between studies. For example, a study that examines eating frequency comparing eating one or two times per $\mathrm{d} v$. four to six times per $\mathrm{d}$ is also encapsulating meal skipping and meal patterns with snacks, respectively. That is, depending on cultural norms, an individual who only eats one or two times per $\mathrm{d}$ may also be considered to be skipping one or two EO. Categorising individuals as being high snack consumers may include individuals who consume snacks in lieu of meals, and therefore future research should consider eating frequency and/or snack frequency in the context of meal frequency/skipping to better differentiate the impact of different types of meal patterns. Some evidence $^{(80)}$ also suggests that healthy and unhealthy dietary patterns can exist among individuals who are highfrequency snack consumers. This may also partially explain the lack of consistent findings for the association between snack frequency and nutrient intakes, and therefore future research should consider examining meal patterns in the context of a individual's overall dietary pattern. Measures of eating frequency may include beverage-only occasions, however, these types of EO have not always been considered when examining the relationship between meal patterns and nutritional intake. This may be an important consideration given the emerging evidence of sugar-sweetened beverages (SSB) in the aetiology of obesity ${ }^{(102,103)}$ and cardiometabolic risk ${ }^{(104,105)}$. Moreover, beverage-only occasions may be especially relevant among certain subgroups of the population; for example, adolescent and young adult males have been shown to be high consumers of $\mathrm{SSB}^{(106)}$.

A limitation of the literature to date on diet quality is that the primary purpose of many of the included studies was to examine associations between meal patterns and health outcomes. Therefore, few of these studies adjusted associations for total EI and important sociodemographic and lifestyle factors. Another limitation of these studies was that meal patterns were often assessed using simple questionnaires with unreported reliability or validity. Importantly, questions regarding meals in questionnaires may not be well defined and this may extend to how respondents should consider beverages.

Under-reporting of EI is a common and well-known limitation of studies that assess dietary intakes ${ }^{(107)}$. Despite this, very few studies on meal patterns have examined the impact of energy misreporting. As eating frequency is positively related to EI, it may be that those who under-report EI also under-report their eating frequency ${ }^{(45,108)}$. There is also some evidence that snacks are more prone to being under-reported $^{(108)}$. Results from a pooled analysis of five large validation studies showed that under-reporting of EI with a single $24 \mathrm{~h}$ recall was approximately $15 \%{ }^{(109)}$. Unless adjusted for, energy misreporting may obscure important relationships between meal patterns, nutrient intakes/diet quality and, ultimately, health outcomes ${ }^{(48)}$. 


\section{Recommendations to advance the field}

To advance the area of meal pattern research, the methods used to collect meal pattern data require further development. Measures that are inexpensive to administer and have low participant burden (for example, questionnaire items) need to be developed and tested for reliability and validity. Contextual information is not always collected as part of a $24 \mathrm{~h}$ recall, yet additional questions about eating location and activities while eating ${ }^{(110)}$ could be considered in order to better understand the contextual factors that influence associations between meal patterns and diet quality. While specific food records have been adapted to collect contextual information (for example, the Weekly Food Diary method ${ }^{(58)}$ ), this method also involves a high participant burden. Dietary assessment methods that utilise new technology (for example, smartphones) may assist in the development of meal pattern research. Devices that people use and carry alongside them every day with the added capacity of a personal digital assistant used in a previous study ${ }^{(32)}$ may be a low burden and efficient way to collect meal pattern data in 'real time'. A major advantage of such technology would be that researchers could collect information allowing examination of all three meal pattern constructs: patterning, format and context. Furthermore, rich contextual data collected in real time could provide insight into the factors that influence participants' decisions to classify an $\mathrm{EO}$ as a meal or snack and therefore help in refining existing meal definitions. Currently little research has examined meal format; however, understanding how different combinations of foods in a meal influence overall diet quality could be an important step in developing a meals-based framework for dietary guidelines. Further work is also required in developing and applying innovative statistical techniques to examining meal patterns, with few applications tested in the literature.

However, it is important to acknowledge that developing new methods to collect and analyse meal patterns data will take considerable time. A major issue still remains of the different definitions available to researchers when conducting meal patterns research. Further analysis (for example, sensitivity analysis) that examines more than one definition from the current literature would facilitate understanding of how the choice of definition makes an impact on the characterisation of meal patterns and associations with outcomes such as nutrient intake and diet quality.

\section{Conclusion}

Overall, there are a number of gaps and limitations in meal pattern research that need to be addressed to further our understanding of how meal definitions influence the characterisation of meal patterns, and the contribution of different meal patterns to nutrient intake and overall diet quality. While current evidence suggests breakfast skipping may be detrimental to diet quality, the nutritional impact of eating frequency, skipping meals other than breakfast and meal timing is inconclusive and warrants further investigation. Future studies should consider how different contexts, beverage-only occasions and energy misreporting affect the relationship between meal patterns and diet quality. The heterogeneity of meal definitions is a major impediment to the interpretation of findings across studies in this field of research. Future research that examines the influence of different meal definitions on the characterisation of meal patterns will facilitate the interpretation of the existing literature, and provide recommendations on the most appropriate methods to advance the field.

\section{Acknowledgements}

R. M. L. is supported by an Australian Postgraduate Award Scholarship. S. A. M. is supported by an Australian Research Council (ARC) Future Fellowship (FT100100581). A. T. is supported by a National Heart Foundation of Australia Future Leader Fellowship (award no. 100046).

R. M. L. drafted the manuscript. S. A. M., A. W. and A. T. provided supervision and critical revision of the manuscript. All authors contributed to and approved the final version of the manuscript.

All authors declare no conflicts of interest.

\section{References}

1. World Health Organization (2009) Global Health Risks: Mortality and Burden of Disease Attributable to Selected Major Risk Factors. Geneva: WHO.

2. Hu FB (2002) Dietary pattern analysis: a new direction in nutritional epidemiology. Curr Opin Lipidol 13, 3-9.

3. McNaughton SA (2010) Dietary patterns and diet quality: approaches to assessing complex exposures in nutrition. Australas Epidemiol 17, 35-37.

4. National Health and Medical Research Council (2013) Australian Dietary Guidelines. Canberra: National Health and Medical Research Council.

5. Mesas AE, Munoz-Pareja M, Lopez-Garcia E, et al. (2012) Selected eating behaviours and excess body weight: a systematic review. Obes Rev 13, 106-135.

6. Szajewska H \& Ruszczynski M (2010) Systematic review demonstrating that breakfast consumption influences body weight outcomes in children and adolescents in Europe. Crit Rev Food Sci Nutr 50, 113-119.

7. Gregori D, Foltran F, Ghidina M, et al. (2011) Understanding the influence of the snack definition on the association between snacking and obesity: a review. Int J Food Sci Nutr 62, 270-275.

8. Johnson GH \& Anderson GH (2010) Snacking definitions: impact on interpretation of the literature and dietary recommendations. Crit Rev Food Sci Nutr 50, 848-871.

9. Bellisle F (2014) Meals and snacking, diet quality and energy balance. Physiol Behav 134, 38-43.

10. Meiselman HL (2009) Dimensions of the meal: a summary. In Meals in Science and Practice: Interdisciplinary Research 
and Business Applications, [HL Meiselman, editor]. Boca Raton, FL: CRC Press, Woodhead Publishing Ltd.

11. Mäkelä J, Kjaernes U, Pipping Ekström M, et al. (1999) Nordic meals: methodological notes on a comparative survey. Appetite 32, 73-79.

12. Bisogni CA, Falk LW, Madore E, et al. (2007) Dimensions of everyday eating and drinking episodes. Appetite $\mathbf{4 8}$, 218-231.

13. Mattes RD (2008) Food palatability, rheology, and meal patterning. JPEN J Parenter Enteral Nutr 32, 572-574.

14. Popkin BM \& Duffey KJ (2010) Does hunger and satiety drive eating anymore? Increasing eating occasions and decreasing time between eating occasions in the United States. Am J Clin Nutr 91, 1342-1347.

15. Bellisle F, Dalix AM, Mennen L, et al. (2003) Contribution of snacks and meals in the diet of French adults: a diet-diary study. Physiol Behav 79, 183-189.

16. Bertéus Forslund H, Lindroos AK, Sjöström L, et al. (2002) Meal patterns and obesity in Swedish women: a simple instrument describing usual meal types, frequency and temporal distribution. Eur J Clin Nutr 56, 740-747.

17. Mekary RA, Giovannucci E, Willett WC, et al. (2012) Eating patterns and type 2 diabetes risk in men: breakfast omission, eating frequency, and snacking. Am J Clin Nutr $\mathbf{9 5}$ 1182-1189.

18. Farshchi HR, Taylor MA \& Macdonald IA (2004) Regular meal frequency creates more appropriate insulin sensitivity and lipid profiles compared with irregular meal frequency in healthy lean women. Eur J Clin Nutr 58, 1071-1077.

19. Sierra-Johnson J, Unden AL, Linestrand M, et al. (2008) Eating meals irregularly: a novel environmental risk factor for the metabolic syndrome. Obesity (Silver Spring) 16, 1302-1307.

20. Jääskeläinen A, Schwab U, Kolehmainen M, et al. (2013) Associations of meal frequency and breakfast with obesity and metabolic syndrome traits in adolescents of Northern Finland Birth Cohort 1986. Nutr Metab Cardiovasc Dis 23 1002-1009.

21. Albertson AM, Franko DL, Thompson D, et al. (2007) Longitudinal patterns of breakfast eating in black and white adolescent girls. Obesity 15, 2282-2292.

22. Smith KJ, Gall SL, McNaughton SA, et al. (2010) Skipping breakfast: longitudinal associations with cardiometabolic risk factors in the Childhood Determinants of Adult Health Study. Am J Clin Nutr 92, 1316-1325.

23. Deshmukh-Taskar PR, Radcliffe JD, Liu Y, et al. (2010) Do breakfast skipping and breakfast type affect energy intake, nutrient intake, nutrient adequacy, and diet quality in young adults? NHANES 1999-2002. J Am Coll Nutr 29, 407-418.

24. Garaulet M, Gómez-Abellán P, Alburquerque-Béjar JJ, et al. (2013) Timing of food intake predicts weight loss effectiveness. Int J Obes (Lond) 37, 604-611.

25. Jakubowicz D, Froy O, Wainstein J, et al. (2012) Meal timing and composition influence ghrelin levels, appetite scores and weight loss maintenance in overweight and obese adults. Steroids $\mathbf{7 7}, 323-331$.

26. Morgan LM, Shi J-W, Hampton SM, et al. (2012) Effect of meal timing and glycaemic index on glucose control and insulin secretion in healthy volunteers. Br J Nutr 108 $1286-1291$.

27. Wang JB, Patterson RE, Ang A, et al. (2014) Timing of energy intake during the day is associated with the risk of obesity in adults. J Hum Nutr Diet 27, Suppl. 2, $255-262$.

28. Cahill LE, Chiuve SE, Mekary RA, et al. (2013) Prospective study of breakfast eating and incident coronary heart disease in a cohort of male US health professionals. Circulation 128, 337-343.

29. Hearty AP \& Gibney MJ (2008) Analysis of meal patterns with the use of supervised data mining techniques - artificial neural networks and decision trees. Am J Clin Nutr $\mathbf{8 8}$, $1632-1642$

30. Kearney JM, Hulshof KF \& Gibney MJ (2001) Eating patterns - temporal distribution, converging and diverging foods, meals eaten inside and outside of the home - implications for developing FBDG. Public Health Nutr 4, 693-698.

31. de Castro JM \& Elmore DK (1988) Subjective hunger relationships with meal patterns in the spontaneous feeding behavior of humans: evidence for a causal connection. Physiol Behav 43, 159-165.

32. Laska MN, Graham D, Moe SG, et al. (2011) Situational characteristics of young adults' eating occasions: a realtime data collection using personal digital assistants. Public Health Nutr 14, 472-479.

33. de Castro JM \& de Castro ES (1989) Spontaneous meal patterns of humans: influence of the presence of other people. Am J Clin Nutr 50, 237-247.

34. Mak TN, Prynne CJ, Cole D, et al. (2012) Assessing eating context and fruit and vegetable consumption in children: new methods using food diaries in the UK National Diet and Nutrition Survey Rolling Programme. Int J Behav Nutr Phys Act 9, 126.

35. Almoosawi S, Winter J, Prynne CJ, et al. (2012) Daily profiles of energy and nutrient intakes: are eating profiles changing over time? Eur J Clin Nutr 66, 678-686.

36. Summerbell CD, Moody RC, Shanks J, et al. (1995) Sources of energy from meals versus snacks in 220 people in four age groups. Eur J Clin Nutr 49, 33-41.

37. Siega-Riz AM, Carson T \& Popkin B (1998) Three squares or mostly snacks - what do teens really eat? A sociodemographic study of meal patterns. $J$ Adolesc Health 22, 29-36.

38. Lennernäs M \& Andersson I (1999) Food-based classification of eating episodes (FBCE). Appetite 32, 53-65.

39. Macdiarmid J, Loe J, Craig LC, et al. (2009) Meal and snacking patterns of school-aged children in Scotland. Eur J Clin Nutr 63, 1297-1304.

40. Drummond SE, Crombie NE, Cursiter MC, et al. (1998) Evidence that eating frequency is inversely related to body weight status in male, but not female, non-obese adults reporting valid dietary intakes. Int $J$ Obes Relat Metab Disord 22, 105-112.

41. Duval K, Strychar I, Cyr MJ, et al. (2008) Physical activity is a confounding factor of the relation between eating frequency and body composition. Am J Clin Nutr $\mathbf{8 8}$ $1200-1205$

42. Ma Y, Bertone ER, Stanek EJ III, et al. (2003) Association between eating patterns and obesity in a free-living US adult population. Am J Epidemiol 158, 85-92.

43. Duffey KJ, Pereira RA \& Popkin BM (2013) Prevalence and energy intake from snacking in Brazil: analysis of the first nationwide individual survey. Eur J Clin Nutr 67, 868-874.

44. Gatenby SJ (1997) Eating frequency: methodological and dietary aspects. Br J Nutr 77, Suppl. 1, S7-S20.

45. Berteus Forslund H, Torgerson JS, Sjostrom L, et al. (2005) Snacking frequency in relation to energy intake and food choices in obese men and women compared to a reference population. Int J Obes (Lond) 29, 711-719.

46. Hampl JS, Heaton CL \& Taylor CA (2003) Snacking patterns influence energy and nutrient intakes but not body mass index. J Hum Nutr Diet 16, 3-11. 
47. Ovaskainen ML, Reinivuo H, Tapanainen H, et al. (2006) Snacks as an element of energy intake and food consumption. Eur J Clin Nutr 60, 494-501.

48. Howarth NC, Huang TT, Roberts SB, et al. (2007) Eating patterns and dietary composition in relation to BMI in younger and older adults. Int J Obes (Lond) 31, 675-684.

49. Chamontin A, Pretzer G \& Booth DA (2003) Ambiguity of 'snack' in British usage. Appetite 41, 21-29.

50. Piernas C \& Popkin BM (2010) Snacking increased among U.S. adults between 1977 and 2006. J Nutr 140, 325-332.

51. Summerbell CD, Moody RC, Shanks J, et al. (1996) Relationship between feeding pattern and body mass index in 220 free-living people in four age groups. Eur J Clin Nutr 50, 513-519.

52. Kerver JM, Yang EJ, Obayashi S, et al. (2006) Meal and snack patterns are associated with dietary intake of energy and nutrients in US adults. J Am Diet Assoc 106, 46-53.

53. Ovaskainen ML, Tapanainen H \& Pakkala H (2010) Changes in the contribution of snacks to the daily energy intake of Finnish adults. Appetite 54, 623-626.

54. Gibney MJ \& Wolever TM (1997) Periodicity of eating and human health: present perspective and future directions. Br J Nutr 77, Suppl. 1, S3-S5.

55. Murakami K \& Livingstone MB (2014) Eating frequency in relation to body mass index and waist circumference in British adults. Int J Obes (Lond) 38, 1200-1206.

56. Smith KJ, Blizzard L, McNaughton SA, et al. (2012) Daily eating frequency and cardiometabolic risk factors in young Australian adults: cross-sectional analyses. $\mathrm{Br} J \mathrm{Nutr}$ 108, 1086-1094.

57. Zizza C, Siega-Riz AM \& Popkin BM (2001) Significant increase in young adults' snacking between 1977-1978 and 1994-1996 represents a cause for concern! Prev Med 32, 303-310

58. de Castro JM (1987) Macronutrient relationships with meal patterns and mood in the spontaneous feeding behavior of humans. Physiol Behav 39, 561-569.

59. Ngo J, Engelen A, Molag M, et al. (2009) A review of the use of information and communication technologies for dietary assessment. Br J Nutr 101, Suppl. 2, S102-S112.

60. Gibson RS (2005) Principles of Nutritional Assessment, 2nd ed.. New York: Oxford University Press.

61. McCrory MA \& Campbell WW (2011) Effects of eating frequency, snacking, and breakfast skipping on energy regulation: symposium overview. J Nutr 141, 144-147.

62. Wirt A \& Collins CE (2009) Diet quality - what is it and does it matter? Public Health Nutr 12, 2473-2492.

63. Kant AK (1996) Indexes of overall diet quality: a review. J Am Diet Assoc 96, 785-791.

64. Min C, Noh H, Kang YS, et al. (2011) Skipping breakfast is associated with diet quality and metabolic syndrome risk factors of adults. Nutr Res Pract 5, 455-463.

65. Roos E \& Prättälä R (1997) Meal pattern and nutrient intake among adult Finns. Appetite 29, 11-24.

66. Barr SI, DiFrancesco L \& Fulgoni VL III (2013) Consumption of breakfast and the type of breakfast consumed are positively associated with nutrient intakes and adequacy of Canadian adults. J Nutr 143, 86-92.

67. Basdevant A, Craplet C \& Guy-Grand B (1993) Snacking patterns in obese French women. Appetite 21, 17-23.

68. Nicklas TA, Myers L, Reger C, et al. (1998) Impact of breakfast consumption on nutritional adequacy of the diets of young adults in Bogalusa, Louisiana: ethnic and gender contrasts. J Am Diet Assoc 98, 1432-1438.

69. Williams P (2005) Breakfast and the diets of Australian adults: an analysis of data from the 1995 National Nutrition Survey. Int J Food Sci Nutr 56, 65-79.
70. Zizza CA, Arsiwalla DD \& Ellison KJ (2010) Contribution of snacking to older adults' vitamin, carotenoid, and mineral intakes. J Am Diet Assoc 110, 768-772.

71. Kuroda T, Onoe Y, Yoshikata R, et al. (2013) Relationship between skipping breakfast and bone mineral density in young Japanese women. Asia Pac J Clin Nutr 22, 583-589.

72. Coates AO, Potter JD, Caan BJ, et al. (2002) Eating frequency and the risk of colon cancer. Nutr Cancer 43, $121-126$

73. Berner LA, Becker G, Wise M, et al. (2013) Characterization of dietary protein among older adults in the United States: amount, animal sources, and meal patterns. I Acad Nutr Diet 113, 809-815.

74. Deshmukh-Taskar PR, Nicklas TA, O'Neil CE, et al. (2010) The relationship of breakfast skipping and type of breakfast consumption with nutrient intake and weight status in children and adolescents: the National Health and Nutrition Examination Survey 1999-2006. J Am Diet Assoc 110, 869-878.

75. Khan MA \& Lipke LK (1982) Snacking and its contribution to food and nutrient intake of college students. J Am Diet Assoc 81, 583-587.

76. Kant AK, Schatzkin A \& Ballard-Barbash R (1997) Evening eating and subsequent long-term weight change in a national cohort. Int J Obes Relat Metab Disord 21, 407-412.

77. Zizza CA, Tayie FA \& Lino M (2007) Benefits of snacking in older Americans. J Am Diet Assoc 107, 800-806.

78. Edelstein SL, Barrett-Connor EL, Wingard DL, et al. (1992) Increased meal frequency associated with decreased cholesterol concentrations; Rancho Bernardo, CA, 19841987. Am J Clin Nutr 55, 664-669.

79. de Castro JM (2004) The time of day of food intake influences overall intake in humans. J Nutr 134, 104-111.

80. Holmbäck I, Ericson U, Gullberg B, et al. (2010) A high eating frequency is associated with an overall healthy lifestyle in middle-aged men and women and reduced likelihood of general and central obesity in men. $\mathrm{Br} J$ Nutr 104, 1065-1073.

81. Winkler G, Döring A \& Keil U (1999) Meal patterns in middle-aged men in Southern Germany: results from the MONICA Augsburg dietary survey 1984/85. Appetite 32, $33-37$.

82. Titan SM, Bingham S, Welch A, et al. (2001) Frequency of eating and concentrations of serum cholesterol in the Norfolk population of the European Prospective Investigation into Cancer (EPIC-Norfolk): cross sectional study. BMJ 323, 1286-1288.

83. Kim SY \& Kim SM (2010) Energy intake and snack choice by the meal patterns of employed people. Nutr Res Pract 4, 43-50.

84. Dattilo M, Crispim CA, Zimberg IZ, et al. (2011) Meal distribution across the day and its relationship with body composition. Biol Rhythm Res 42, 119-129.

85. Mekary RA, Hu FB, Willett WC, et al. (2012) The joint association of eating frequency and diet quality with colorectal cancer risk in the Health Professionals Follow-up Study. Am J Epidemiol 175, 664-672.

86. Mekary RA, Giovannucci E, Cahill L, et al. (2013) Eating patterns and type 2 diabetes risk in older women: breakfast consumption and eating frequency. Am J Clin Nutr 98, 436-443

87. Odegaard AO, Jacobs DR Jr, Steffen LM, et al. (2013) Breakfast frequency and development of metabolic risk. Diabetes Care 36, 3100-3106.

88. Kim S, DeRoo LA \& Sandler DP (2011) Eating patterns and nutritional characteristics associated with sleep duration. Public Health Nutr 14, 889-895. 
89. Zizza CA \& Xu B (2012) Snacking is associated with overall diet quality among adults. J Acad Nutr Diet 112, 291-296.

90. Smith KJ, McNaughton SA, Cleland VJ, et al. (2013) Health, behavioral, cognitive, and social correlates of breakfast skipping among women living in socioeconomically disadvantaged neighborhoods. J Nutr 143, 1774-1784.

91. Dewolfe J \& Millan K (2003) Dietary intake of older adults in the Kingston area. Can J Diet Pract Res 64, 16-24.

92. Shatenstein B, Gauvin L, Keller H, et al. (2013) Baseline determinants of global diet quality in older men and women from the NuAge cohort. J Nutr Health Aging 17, 419-425.

93. Mesas AE, Guallar-Castillon P, Leon-Munoz LM, et al. (2012) Obesity-related eating behaviors are associated with low physical activity and poor diet quality in Spain. $J$ Nutr 142, 1321-1328.

94. Azadbakht L, Haghighatdoost F, Feizi A, et al. (2013) Breakfast eating pattern and its association with dietary quality indices and anthropometric measurements in young women in Isfahan. Nutrition 29, 420-425.

95. Wansink B, Payne CR \& Shimizu M (2010) Is this a meal or snack? Situational cues that drive perceptions. Appetite $\mathbf{5 4}$, $214-216$

96. Kant AK, Graubard BI \& Mattes RD (2012) Association of food form with self-reported 24-h energy intake and meal patterns in US adults: NHANES 2003-2008. Am J Clin Nutr 96, 1369-1378.

97. Schwarz N (1999) Self-reports: how the questions shape the answers. Am Psychol 54, 93-105.

98. Oltersdorf U, Schlettwein-Gsell D \& Winkler G (1999) Assessing eating patterns - an emerging research topic in nutritional sciences: introduction to the symposium. Appetite 32, 1-7.

99. Almoosawi S, Prynne CJ, Hardy R, et al. (2013) Time-of-day of energy intake: association with hypertension and blood pressure 10 years later in the 1946 British Birth Cohort. J Hypertens 31, 882-892.
100. Almoosawi S, Prynne CJ, Hardy R, et al. (2013) Time-of-day and nutrient composition of eating occasions: prospective association with the metabolic syndrome in the 1946 British Birth Cohort. Int J Obes 37, 725-731.

101. Chapelot D (2011) The role of snacking in energy balance: a biobehavioral approach. J Nutr 141, 158-162.

102. Malik VS, Schulze MB \& Hu FB (2006) Intake of sugarsweetened beverages and weight gain: a systematic review. Am J Clin Nutr 84, 274-288.

103. Malik VS, Pan A, Willett WC, et al. (2013) Sugar-sweetened beverages and weight gain in children and adults: a systematic review and meta-analysis. Am J Clin Nutr $\mathbf{9 8}$, 1084-1102.

104. Bhupathiraju SN, Pan A, Malik VS, et al. (2013) Caffeinated and caffeine-free beverages and risk of type 2 diabetes. Am J Clin Nutr 97, 155-166.

105. Ambrosini GL, Oddy WH, Huang RC, et al. (2013) Prospective associations between sugar-sweetened beverage intakes and cardiometabolic risk factors in adolescents. Am J Clin Nutr 98, 327-334.

106. Australian Bureau of Statistics (2014) Soft Drink, Burgers and Chips - The Diet of Our Youth. Australian Health Survey: Nutrition First Results - Foods and Nutrients, 2011-12. Canberra: ABS, Cat. no. 4364.0.55.007.

107. Livingstone MB \& Black AE (2003) Markers of the validity of reported energy intake. J Nutr 133, Suppl. 3, 895S-920S

108. Bellisle F, McDevitt R \& Prentice AM (1997) Meal frequency and energy balance. Br J Nutr 77, Suppl. 1, S57-S70.

109. Freedman LS, Commins JM, Moler JE, et al. (2014) Pooled results from 5 validation studies of dietary self-report instruments using recovery biomarkers for energy and protein intake. Am J Epidemiol 180, 172-188.

110. Subar AF, Kirkpatrick SI, Mittl B, et al. (2012) The Automated Self-Administered 24-hour dietary recall (ASA24): a resource for researchers, clinicians, and educators from the National Cancer Institute. J Acad Nutr Diet 112, $1134-1137$. 